\title{
A QUADRUPOLAR PREPLANETARY NEBULA: IRAS 19475+3119
}

\author{
Raghvendra Sahai, ${ }^{1}$ Carmen Sánchez Contreras, ${ }^{2}$ Mark Morris, ${ }^{3}$ and Mark Claussen ${ }^{4}$ \\ Received 2006 September 14; accepted 2006 November 24
}

\begin{abstract}
Our imaging of the preplanetary nebula IRAS $19475+3119$ (hereafter I19475) at 0.43 and $0.6 \mu \mathrm{m}$ with HST reveals a quadrupolar nebula of size about $10.5^{\prime \prime} \times 4.7^{\prime \prime}$, with two bipolar elongated lobes emanating from the center of the nebula. One of the bipolar lobes shows detailed point-symmetric structure with respect to the central star. A faint, surface brightness-limited, diffuse halo surrounds the lobes. Interferometric observations of the $\mathrm{CO} J=1-0$ line with OVRO at $\sim 8^{\prime \prime}$ resolution show that the bulk of the emission comes from an unresolved molecular envelope expanding at $15 \mathrm{~km} \mathrm{~s}^{-1}$, resulting from the AGB progenitor's dense, slow wind. Weaker emission is seen from a fast bipolar outflow oriented along the longer and more tenuous pair of the sets of lobes. Optical spectroscopy reveals a complex $\mathrm{H} \alpha$ profile with a broad photospheric absorption feature and a narrow inverse $\mathrm{P}$ Cygni shaped core; comparison with previous data shows that the core profile shape varies dramatically with time. We find an unresolved source of millimeter-wave continuum emission in I19475, which together with previous submillimeter continuum measurements, implies a very substantial mass (roughly a few times $0.01 M_{\odot}$ ) of large (radius $\gtrsim 1 \mathrm{~mm}$ ), cold $(\sim 30 \mathrm{~K})$ dust grains in I19475. Combining our estimates of the circumstellar mass with the typical mass of a post-AGB star, we find that the main-sequence progenitor of I19475's central star had a mass $\gtrsim 2.5 M_{\odot}$. We discuss the formation of I19475's quadrupolar nebula in the light of past and current ideas for the dramatic transformation of the morphology and kinematics of mass ejecta as AGB stars evolve into planetary nebulae.
\end{abstract}

Subject headings: circumstellar matter — dust, extinction — planetary nebulae: general -

stars: AGB and post-AGB — stars: mass loss — stars: individual (IRAS 19475+3119)

Online material: color figures

\section{INTRODUCTION}

Preplanetary nebulae (PPNs), transition objects between the asymptotic giant branch (AGB) and planetary nebula (PN) phases, probably hold the key to understanding how the slowly expanding $\left(5-15 \mathrm{~km} \mathrm{~s}^{-1}\right)$, largely spherical, circumstellar envelopes (CSEs) of AGB stars transform into highly aspherical PNs with fast outflows $\left(\gtrsim 100 \mathrm{~km} \mathrm{~s}^{-1}\right)$ along one or more axes (e.g., Sahai \& Trauger 1998; Balick \& Frank 2002). We are therefore carrying out a program of multiwavelength imaging and spectroscopic observations of young PPNs, using a large ( 300), morphologically unbiased sample (Sahai \& Sánchez Contreras 2004) mainly constructed from catalogs of OH/IR stars (evolved, visually faint, mass-losing stars with dense circumstellar envelopes, showing generally double-peaked $\mathrm{OH}$ maser emission). Interferometric mapping of the $\mathrm{OH}$ emission in many of these objects (e.g., Bowers et al. 1983) shows that it typically arises in an extended circular shell of radius $\sim 10^{16} \mathrm{~cm}$, indicating that substantial AGB mass loss has occurred through a spherical outflow. Furthermore, a recent decrease in their mass-loss rates, marking the beginning of their post-AGB evolution, is indicated by their 12 to $25 \mu \mathrm{m}$ IRAS flux ratios, which are generally smaller than 1 , implying a lack of dust hotter than about $450 \mathrm{~K}$ and therefore cessation of the dense AGB mass-loss process about 100-200 yr ago.

As part of our multiwavelength program, we have carried out several HST SNAPshot imaging surveys of our young PPN

\footnotetext{
1 Jet Propulsion Laboratory, California Institute of Technology, Pasadena, CA; raghvendra.sahai@jpl.nasa.gov.

2 California Institute of Technology, Pasadena, CA. Current address: Departamento de Astrofisica Molecular e Infraroja, IEM-CSIC, Madrid, Spain.

${ }_{3}^{3}$ Division of Astronomy, Department of Physics and Astrophysics, UCLA, Los Angeles, CA.

${ }^{4}$ National Radio Astronomy Observatory, Socorro, NM.
}

sample - a substantial number of our objects have been resolved and show bipolar or multipolar morphologies (e.g., Sahai 2004). Detailed studies of the best resolved objects are now in progresswe recently reported the discovery of two young PPNs, one an icy, bipolar object with knotty jets (IRAS 22036+5306; Sahai et al. 2003) and another that is multipolar (IRAS 19024+0044; Sahai et al. 2005).

We now present our optical HST imaging of the PPN IRAS $19475+3119$ (hereafter I19475), which was classified as a postAGB object by Hrivnak et al. (1999) based on its double-peaked spectral energy distribution (SED). Optical spectra of its central star, HD 331319, show a spectral type of F3 Ib (Klochkova et al. 2002). Single-dish CO spectra show a strong central core of width $\sim 25 \mathrm{~km} \mathrm{~s}^{-1}$ as well as weak wings, presumably due to emission from the remnant AGB circumstellar envelope and a high-speed outflow, respectively (Likkel et al. 1991; Hrivnak \& Bieging 2005). Bujarrabal et al. (2001) used similar single-dish CO spectra to derive the mass and linear momentum of the high-velocity outflow. Very recently, Sánchez Contreras et al. (2006, hereafter SC06) have carried out high angular resolution $\left(2^{\prime \prime}\right)$ interferometric mapping of the $\mathrm{CO} J=2-1$ emission, revealing the structure of two distinct nebular components, namely, a slowly expanding shell and a fast bipolar outflow. Near-infrared imaging by Gledhill et al. (2001) showed a faint, extended $\left(\sim 4^{\prime \prime}\right)$, asymmetric nebula. Sarkar \& Sahai (2006, hereafter SS06) have made detailed models of the full SED of this object, including its broadband Midcourse Science Experiment (MSX) and Infrared Astronomical Satellite (IRAS) photometry and ISO (Infrared Space Observatory) spectra, and find a strong submillimeter excess, which they interpret as emission arising from large, cool grains.

Our HST imaging of I19475 reveals a quadrupolar nebula. We have also obtained supporting interferometric $\mathrm{CO} J=1-0$ mapping and optical echelle spectroscopic observations. In this 
paper we analyze these data, together with archival infrared and far-infrared data from $M S X$ and IRAS, to determine the physical characteristics of this PPN.

The rest of the paper is organized as follows. Section 2 describes our multiwavelength imaging and spectroscopic observations; $\S 3$ describes the results of these observations; the distance, luminosity, and reddening of the central star are discussed in $\S 4$; the HST images are analyzed in $\S 5$ to derive the physical characteristics of individual nebular structures; in $\S 6$ we analyze the $\mathrm{CO}$ data to derive the physical properties of the molecular shell and the ${ }^{13} \mathrm{C} /{ }^{12} \mathrm{C}$ isotope ratio; in $\S 7$ we refine the existing dust model to fit our new millimeter-wave and existing submillimeter-wave data; in $\S 8$ the structure and formation of I19475 is discussed in the context of theoretical models, and finally, our main conclusions are summarized in $\S 9$.

\section{OBSERVATIONS}

\subsection{Optical Imaging with HST}

I19475 was imaged on UT date 2003 November 1 (GO program 9463), by the High Resolution Camera (HRC) of the Advanced Camera for Surveys (ACS), which has a plate scale of $0.025^{\prime \prime} \mathrm{pixel}^{-1}$, using a Johnson $B$ filter $(\mathrm{F} 435 \mathrm{~W})$ with exposures of $3 \times 75,20$, and $0.8 \mathrm{~s}$, and a broad $V$ filter $(\mathrm{F} 606 \mathrm{~W})$ with exposures of $3 \times 7.4,2.0$, and $0.2 \mathrm{~s}$. The longest exposure images for each filter were obtained with a three-point dither. The standard STScI HST pipeline calibration has been applied to all data. We have preserved the intrinsic orientation of the HRC images in the figures in this paper, electing not to rotate them for alignment of their horizontal and vertical axes with the cardinal directions because such rotation would result in some degradation of the image quality. Several field stars present in the images have been used to achieve satisfactory registration between the dithered images.

\subsection{Long-Slit Optical Spectroscopy}

Echelle spectra were obtained with the $10 \mathrm{~m}$ Keck II telescope (Mauna Kea, Hawaii) on 2003 June 1 using the Echelle Spectrograph and Imager (ESI). ${ }^{5}$ The detector was a MIT-LL CCD with $2048 \times 4096$ pixels of $15 \mu \mathrm{m}$, and the total wavelength coverage of $\sim 3900-11000 \AA$. The reciprocal dispersion and the pixel angular scale range from 0.15 to $0.39 \AA$ pixel $^{-1}$ and from $0.120^{\prime \prime}$ to $0.168^{\prime \prime}$, respectively, for 10 echelle orders. We used a $0.5^{\prime \prime} \times 20^{\prime \prime}$ slit oriented at P.A. $=-42^{\circ}$ (measured east of north). Total integration time was $2400 \mathrm{~s}$. Data were reduced using IRAF. ${ }^{6}$ We used $\mathrm{CuAr}$ lamps to perform wavelength calibration. The velocity resolution achieved (FWHM of the lamp lines) is $\sim 37 \mathrm{~km} \mathrm{~s}^{-1}$ for all orders. Two photometric standards, LTT 9239 and Feige 110 were used for flux calibration. Seeing was $\sim 0.6^{\prime \prime}$. Weather conditions were nonphotometric during the observations.

\subsection{Millimeter-Wave Interferometry}

Interferometric imaging of the ${ }^{12} \mathrm{CO}$ and ${ }^{13} \mathrm{CO} J=1-0$ emission lines (with rest frequency 115,271.204 MHz and $110,201.36 \mathrm{MHz}$, respectively) of I19475 was carried out us-

\footnotetext{
${ }^{5}$ The W. M. Keck Observatory is operated as a scientific partnership among the California Institute of Technology, the University of California, and the National Aeronautics and Space Administration. The Observatory was made possible by the generous financial support of the W. M. Keck Foundation. The authors wish to recognize and acknowledge the very significant cultural role and reverence that the summit of Mauna Kea has always had within the indigenous Hawaiian community. We are most fortunate to have the opportunity to conduct observations from this mountain.

${ }^{6}$ IRAF is distributed by the National Optical Astronomy Observatory, which is operated by the Association of Universities for Research in Astronomy (AURA), Inc., under cooperative agreement with the National Science Foundation.
}

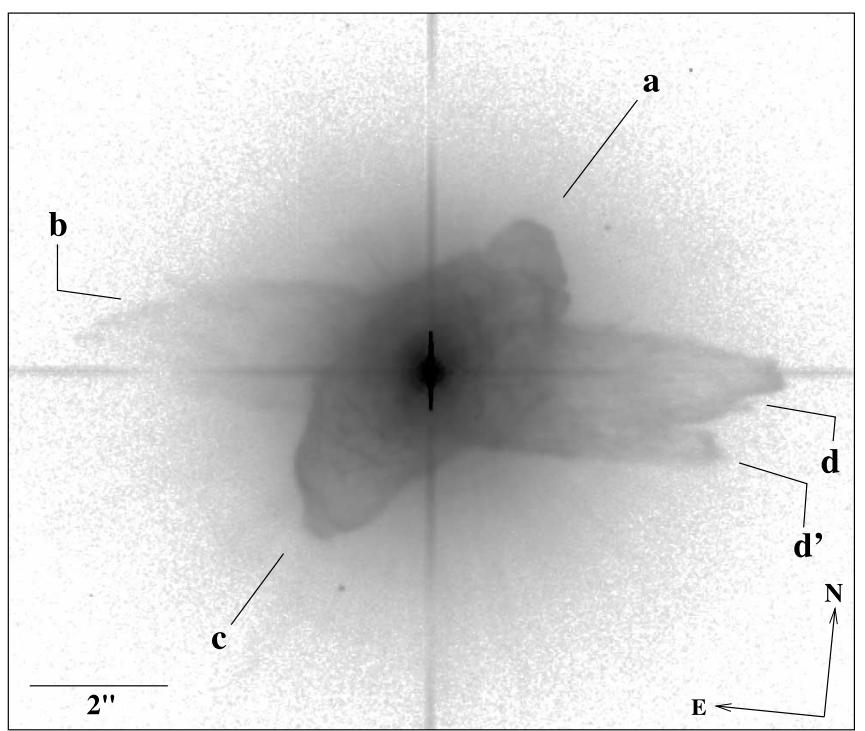

FIG. 1.-Image (log stretch) of the preplanetary nebula IRAS 19475+3119 made using exposures taken with the HST ACS through the F435W filter. The maximum (black) and minimum (white) intensities of the gray scale are $6.87 \times$ $10^{-13}$ and $4.33 \times 10^{-17} \mathrm{ergs} \mathrm{s}^{-1} \mathrm{~cm}^{-2} \AA^{-1} \operatorname{arcsec}^{-2}$, respectively. Lobe $\mathrm{d}$ appears to be composite, with a minor lobe, d'. A smooth, round halo surrounds the lobes.

ing the millimeter interferometer of the Owens Valley Radio Observatory (OVRO), which consists of six $10.4 \mathrm{~m}$ diameter antennas. ${ }^{7}$ An SIS receiver fixed tuned in double-sideband mode was used, allowing simultaneous observations of the ${ }^{12} \mathrm{CO}$ and ${ }^{13} \mathrm{CO}$ lines. Observations were performed on 2003 January 29, May 8, and May 22 in low (C) configuration, for which baselines between antennas range from 18 to $55 \mathrm{~m}$. The units of the digital spectral line correlator were arranged to provide a total bandwidth of $90 \mathrm{MHz}$ (234 and $245 \mathrm{~km} \mathrm{~s}^{-1}$ for the ${ }^{12} \mathrm{CO}$ and ${ }^{13} \mathrm{CO}$ lines, respectively) with a channel spacing of $1 \mathrm{MHz}\left(2.06\right.$ and $2.72 \mathrm{~km} \mathrm{~s}^{-1}$ for the ${ }^{12} \mathrm{CO}$ and ${ }^{13} \mathrm{CO}$ lines, respectively). The $3 \mathrm{~mm}$ continuum emission from I19475 was observed simultaneously using the dualchannel analog continuum correlator, which provided a total bandwidth of $4 \mathrm{GHz}$ after combining both intermediate frequency (IF) bands. Total integration time on source was $\sim 10 \mathrm{hr}$.

The calibration of the data was performed using the MMA software package. ${ }^{8}$ Data were gain calibrated in baseline-based mode using the quasar J2015+371, which was observed at regular time intervals of $\sim 20$ minutes before and after our target. The quasars 3C 345, 3C 273, and 3C 84 were used as passband calibrators. Flux calibration was performed observing Uranus; quasars were also used as secondary flux calibrators.

Reconstruction of the maps from the visibilities was done using the Multichannel Image Reconstruction, Image Analysis, and Display (MIRIAD) software. We Fourier-transformed the measured visibilities with robust weighting, which is an optimized compromise between natural and uniform weighting. For the continuum image we used natural weighting to optimize the signal-tonoise ratio $(\mathrm{S} / \mathrm{N})$. After that, the images were cleaned. The clean beam for our ${ }^{12} \mathrm{CO}\left({ }^{13} \mathrm{CO}\right)$ maps has $\mathrm{FWHM}=9.2^{\prime \prime} \times 6.7^{\prime \prime}$ $\left(10.7^{\prime \prime} \times 8.5^{\prime \prime}\right)$ and is oriented at P.A. $=82^{\circ}\left(79^{\circ}\right)$; the conversion factor from surface brightness to temperature units is $1.5 \mathrm{~K} \mathrm{Jy}^{-1}$ beam $^{-1}\left(1.1 \mathrm{~K} \mathrm{Jy}^{-1}\right.$ beam $\left.^{-1}\right)$. The noise $(1 \sigma)$ in the ${ }^{12} \mathrm{CO}\left({ }^{13} \mathrm{CO}\right)$ maps is $\sim 40(20)$ mJy beam ${ }^{-1}$, as measured in regions with no signal, and the dynamic range is 20 (17). The

\footnotetext{
7 See http://www.ovro.caltech.edu/mm for more details.

${ }^{8}$ MMA is written and maintained by the Caltech Millimeter Interferometry
} 


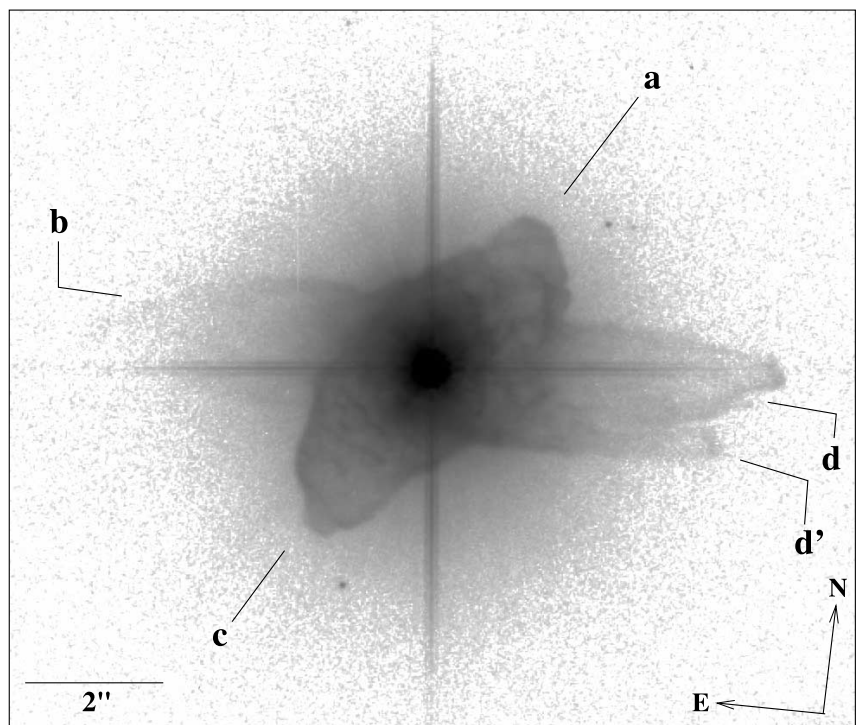

FIG. 2.- Image ( $\log$ stretch) of the preplanetary nebula IRAS $19475+3119$ made using exposures taken with the HST ACS through the F606W filter. The maximum (black) and minimum (white) intensities of the gray scale are $2.04 \times$ $10^{-13}$ and $5.12 \times 10^{-17} \mathrm{ergs} \mathrm{s}^{-1} \mathrm{~cm}^{-2} \AA^{-1} \operatorname{arcsec}^{-2}$, respectively.

clean beam for our continuum map has FWHM $=10.7^{\prime \prime} \times 8.5^{\prime \prime}$ and is oriented at P.A. $=79^{\circ}$, and the conversion factor from surface brightness to temperature units is $1.1 \mathrm{~K} \mathrm{Jy}^{-1}$ beam $^{-1}$. The noise in the continuum map, which was obtained using the continuum correlator bands that were free from line emission (covering a total bandwidth of $3 \mathrm{GHz}$ ), is $\sigma=0.9 \mathrm{mJy} \mathrm{beam}^{-1}$.

\section{RESULTS}

\subsection{Optical Imaging}

Both the F435W and F606W images (Figs. 1-2) show a quadrupolar nebula, with two prominent elongated bipolar lobes emanating from the center of the nebula (labeled " $a-c$ " and " $b-d$ " in Figs. 1 and 2). Lobes $\mathrm{b}-\mathrm{d}(\mathrm{a}-\mathrm{c})$ are aligned roughly along P.A. $=90\left(-55^{\circ}\right)$. Lobe $d$ has a secondary component $d^{\prime}$. The lobes show significant limb brightening, and the shape of the $\mathrm{a}-\mathrm{c}$ lobe pair appears to be point symmetric about the central star. A very faint, presumably surface brightness-limited, diffuse halo surrounds the lobes. The size of the nebula, defined as the smallest rectangle circumscribing the lobes is $\sim 10.5^{\prime \prime} \times 4.7^{\prime \prime}$.

As in the near-infrared (Gledhill et al. 2001), the nebula is seen predominantly in light from the central star, scattered by dust in the lobes. Our optical spectroscopy (see $\S 3.2$ ) shows that the contribution of lines to the broadband F435W and F606W filters is negligible. The limb-brightened appearance of the lobes, suggests that they are dense-walled structures with tenuous interiors. The a-c lobe pair shows complex microstructure, apparently organized in the form of a large number of intersecting loops and filaments (Fig. 3) - similar structure has been found previously in another PPN, Roberts 22 (Sahai et al. 1999). The color of the lobes (Fig. 4) does not change strongly along their length beyond the central region, which supports the idea that the lobes are relatively hollow so that the starlight does not experience an increasing amount of reddening with radius before being scattered by dust in the lobe walls.

Using the photometric calibration keywords in the image headers, we find that the integrated fluxes for the nebula and central star at 0.43 and $0.6 \mu \mathrm{m}$ are $6.5 \times 10^{-13}$ and $6.43 \times 10^{-13} \mathrm{ergs} \mathrm{cm}^{-2}$ $\mathrm{s}^{-1} \AA^{-1}$, respectively, corresponding to $B, V$, and $R$ magnitudes $^{9}$ of $10.0,9.2$, and 8.94. In comparison, the USNO-B. 1 cat$\operatorname{alog}^{10}$ gives $B=10.23(9.88)$ and $R=9.14(9.1)$ for epoch 1 (2),

9 To convert from the fluxes measured in the different filters above to magnitudes, we have used the Units Conversion Tool at http://www.stsci.edu/ $\mathrm{hst} /$ nicmos/tools/conversion_form.html, assuming a $T_{\text {eff }}=7500 \mathrm{~K}$ stellar blackbody to describe the SED (see $\S 4$ ); the results are not very sensitive to the adopted $T_{\text {eff }}$. Since the "broad $V$ " filter F606W has significant overlap with both the $V$ and $R$ Johnson filter bandpasses, we derive both $V$ and $R$ magnitudes from our F606W data.

10 USNO-B.1 name: B1.0 1214-0417041; J2000.0 coordinates: $\alpha=$ $19^{\mathrm{h}} 49^{\mathrm{m}} 29.56^{\mathrm{s}}, \delta=31^{\circ} 27^{\prime} 16.3^{\prime \prime}$.
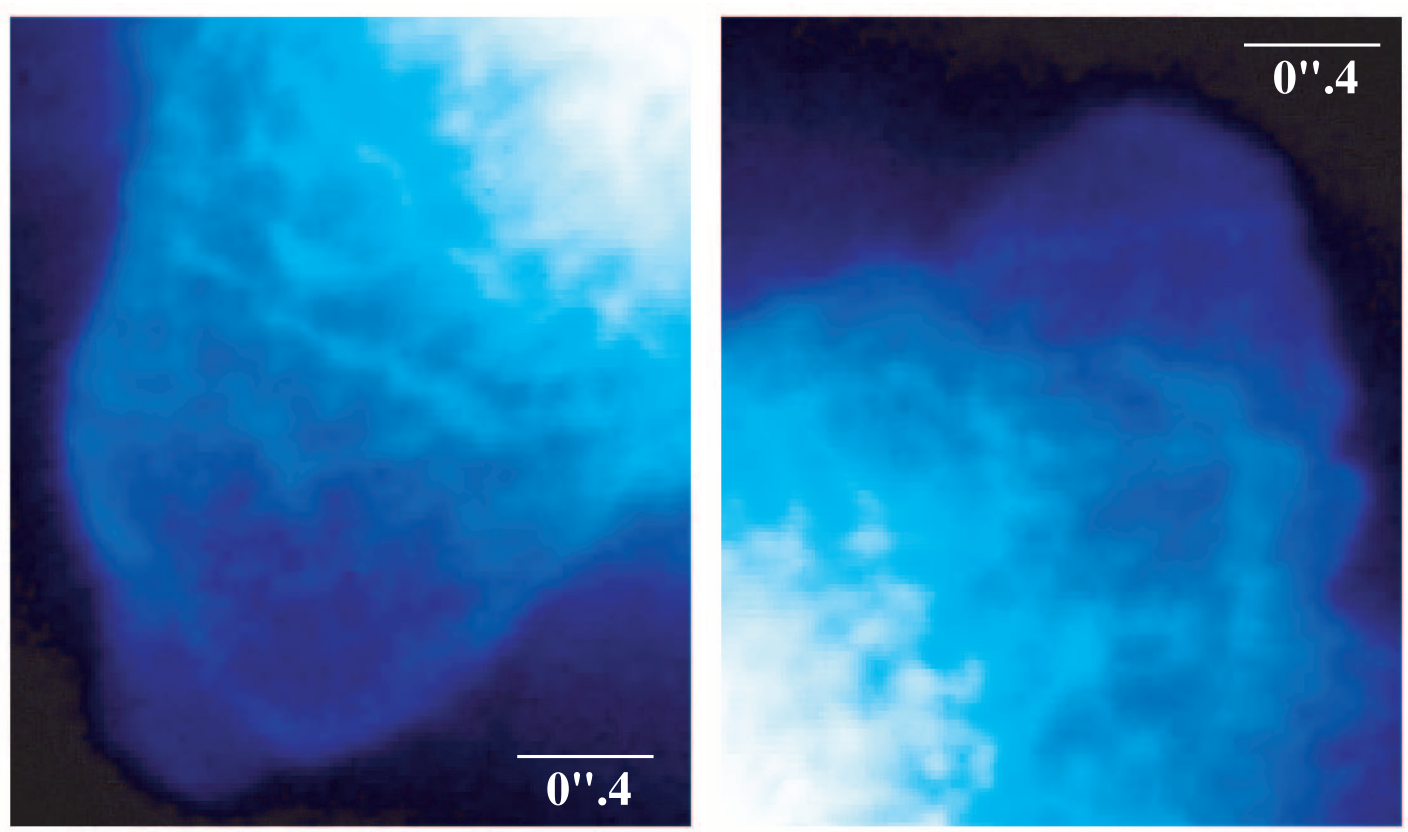

FIG. 3.-False-color image of the $\mathrm{c}(l e f t)$ and a $(r i g h t)$ lobes of IRAS 19475+3119. Sharp structures have been enhanced in these images. The orientation is the same as in Fig. 1. 


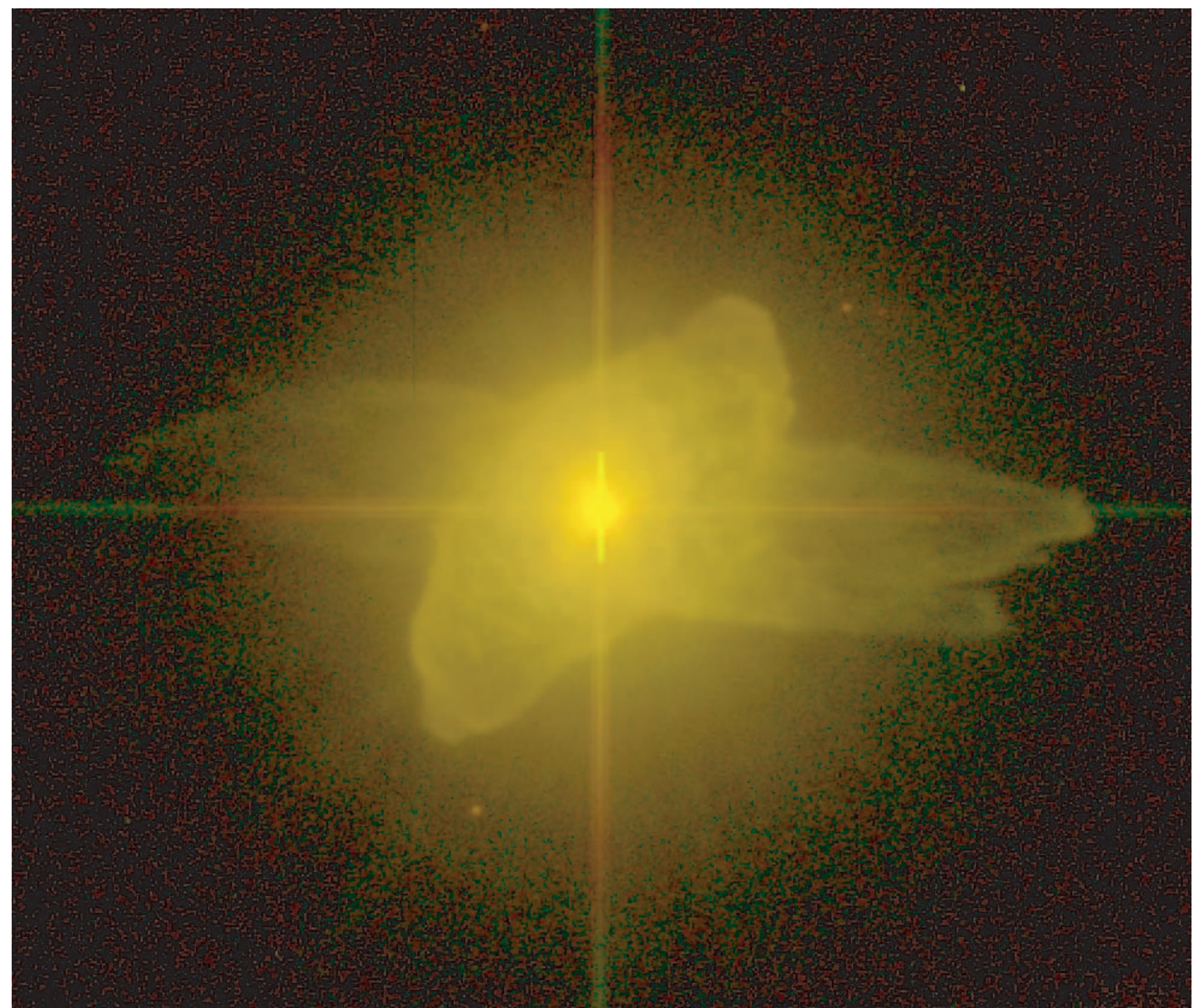

FIG. 4.-False-color image of IRAS 19475+3119 made by combining the F435W and F606W filter images. The scale and orientation are the same as in Fig. 1.

and the GSC 2.2 catalog gives $B=10.35 \pm 0.03$ and $V=$ $9.6 \pm 0.02$.

We do not see the spiral structure reported by Gledhill et al. (2001, their Fig. 16) from their $J$-band polarized flux image. This is surprising considering that the flux seen in their $J$-band image is due to scattered light, because we expect the HST images to be much more sensitive to detecting structures in scattered light due to the much larger scattering cross-sections at the shorter wavelengths. This expectation is supported by our detection of the faint lobes $b$ and d, which are not seen in the $J$-band images. Our $H S T$ images provide a plausible alternative explanation for the spiral structure, which appears to lie in the region of overlap between the $a-c$ and $b-d$ lobe pairs. We think that the spiral structure may not be an intrinsic physical structure in the a-c lobe pair, but rather an artifact resulting from poorly resolved regions of enhanced projected column density where the $a-c$ and $b-d$ lobe pairs overlap along the line of sight.

\subsection{Optical Spectroscopy}

Since high-resolution optical spectroscopy has already been used by Klochkova et al. (2002) to discuss the spectral type, luminosity class, and composition of the central star of I19475, we confine ourselves here to a discussion of the rather unique $\mathrm{H} \alpha$ line profile, which is also time variable. As in Klochkova et al. (2002), our H $\alpha$ profile of I19475 (Fig. 5) shows a wide absorption $(\mathrm{FWHM}=10.5 \AA)$ line, and the peak depth of this feature is about $15 \%$ of the continuum in both spectra. However, in our spectrum there is a very narrow inverse $\mathrm{P}$ Cygni component in the line core (each of the emission and absorption components of this core has an FWHM of $0.84 \AA$ ). In contrast, the spectrum of Klochkova et al. (2002) shows a double-peaked emission core component with a deep central absorption. Klochkova et al. compare their $\mathrm{H} \alpha$ profile for three epochs and find that the intensity of the short-wavelength emission peak increases simultaneously with a decrease in the intensity of the long-wavelength emission peak. Our line-shape thus fits into this pattern, representing the variational extreme in which the long-wavelength peak disappears entirely. Klochkova et al. subtract a model photospheric

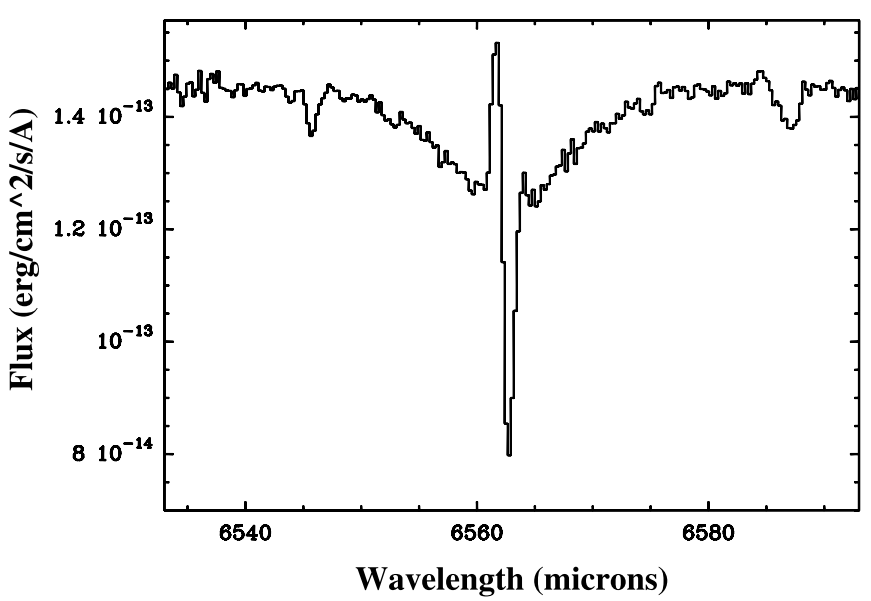

FIG. 5.- $\mathrm{H} \alpha$ line profile of I19475 observed with the Keck ESI instrument. 


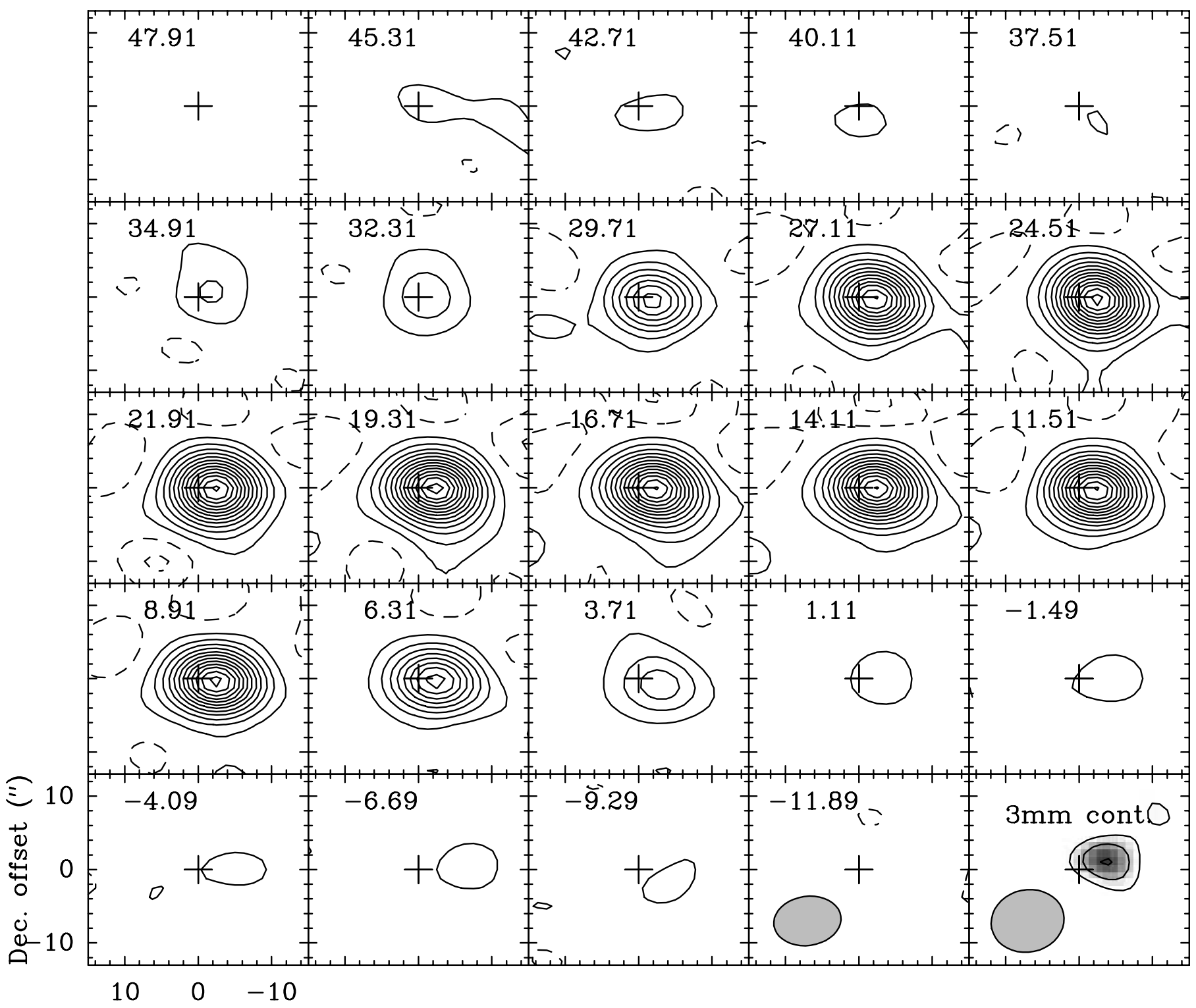

R.A. offset (")

FIG. 6. $-\mathrm{CO} J=1-0$ channel maps and $2.6 \mathrm{~mm}$ continuum map of IRAS $19475+3119$ obtained at OVRO. Each panel for the CO line covers a velocity bin of width $2.6 \mathrm{~km} \mathrm{~s}^{-1}$, centered at the LSR velocity shown near the top left corner. Contour levels are as follows: (1) for the CO line, positive contours run from $2 \sigma$ in $3 \sigma$ steps; and negative contours run from $-2 \sigma$ in $-3 \sigma$ steps, where $\sigma=40 \mathrm{mJy} \mathrm{beam}^{-1}$ is the rms noise; (2) for the continuum contours run from $2 \sigma$ in $1 \sigma$ steps, where $\sigma=0.9$ mJy beam ${ }^{-1}$. The cross is located at the tracking center of the observations $\left(\alpha_{\mathrm{J} 2000.0}=19^{\mathrm{h}} 49^{\mathrm{m}} 29.74^{\mathrm{s}}, \delta_{\mathrm{J} 2000.0}=31^{\circ} 27^{\prime} 16.33^{\prime \prime}\right)$. The peak of the CO emission is located at $\alpha_{\mathrm{J} 2000.0}=$ $19^{\mathrm{h}} 49^{\mathrm{m}} 29.56^{\mathrm{s}}, \delta_{\mathrm{I} 2000.0}=31^{\circ} 27^{\prime} 16.1^{\prime \prime}$.

line profile (using an enhanced He abundance) from their spectra in order to separate the emission component, which they suggest arises in a rotating disk around the central star, as in Be stars. However, no explanation is offered for the time variations of the $\mathrm{H} \alpha$ profile in the context of this model.

\subsection{Millimeter-Wave Observations}

Our interferometric imaging of ${ }^{12} \mathrm{CO}$ and ${ }^{13} \mathrm{CO} J=1-0$ emission toward I19475 is shown in Figures 6 and 7. The integrated line profiles (Fig. 8) are centered at $V_{\mathrm{lsr}}=18 \mathrm{~km} \mathrm{~s}^{-1}$, with an intense central component, which has a full width at zero intensity (FWZI) of $\sim 30 \mathrm{~km} \mathrm{~s}^{-1}$ derived from fitting an empirical profile. The shape of the central component is typical of $\mathrm{CO}$ profiles from the circumstellar envelopes of AGB stars and im- plies the presence of a molecular envelope expanding at $V_{e, \mathrm{AGB}} \sim$ $15 \mathrm{~km} \mathrm{~s}^{-1}$. The higher $\mathrm{S} / \mathrm{N}^{12} \mathrm{CO}$ line profile also shows a weak, broad component with a FWZI of $\sim 63 \mathrm{~km} \mathrm{~s}^{-1}$. The peaks of the red and blue emission in the broad component are spatially separated by about $3.4^{\prime \prime}$ along the long axis of the nebula, i.e., along the axis of lobes $b$ and d (Fig. 9). The blue (red) component appears associated with lobe $\mathrm{d}(\mathrm{b})$, implying that lobe $\mathrm{d}(\mathrm{b})$ is tilted toward (away) from us. Higher resolution mapping $\left(2^{\prime \prime}\right)$ of the ${ }^{12} \mathrm{CO} J=2-1$ line emission from I19475 has been reported by SC06, who find the high-velocity outflow to extend in a 7.5" long structure along the axis of the lobe-pair $\mathrm{b}-\mathrm{d}$. An unresolved central continuum emission source was detected with a flux $F_{3 \mathrm{~mm}}=$ $3.6 \pm 0.8 \mathrm{mJy}$; the error bars only include statistical errors. However, since systematic flux calibration errors are not expected to be 


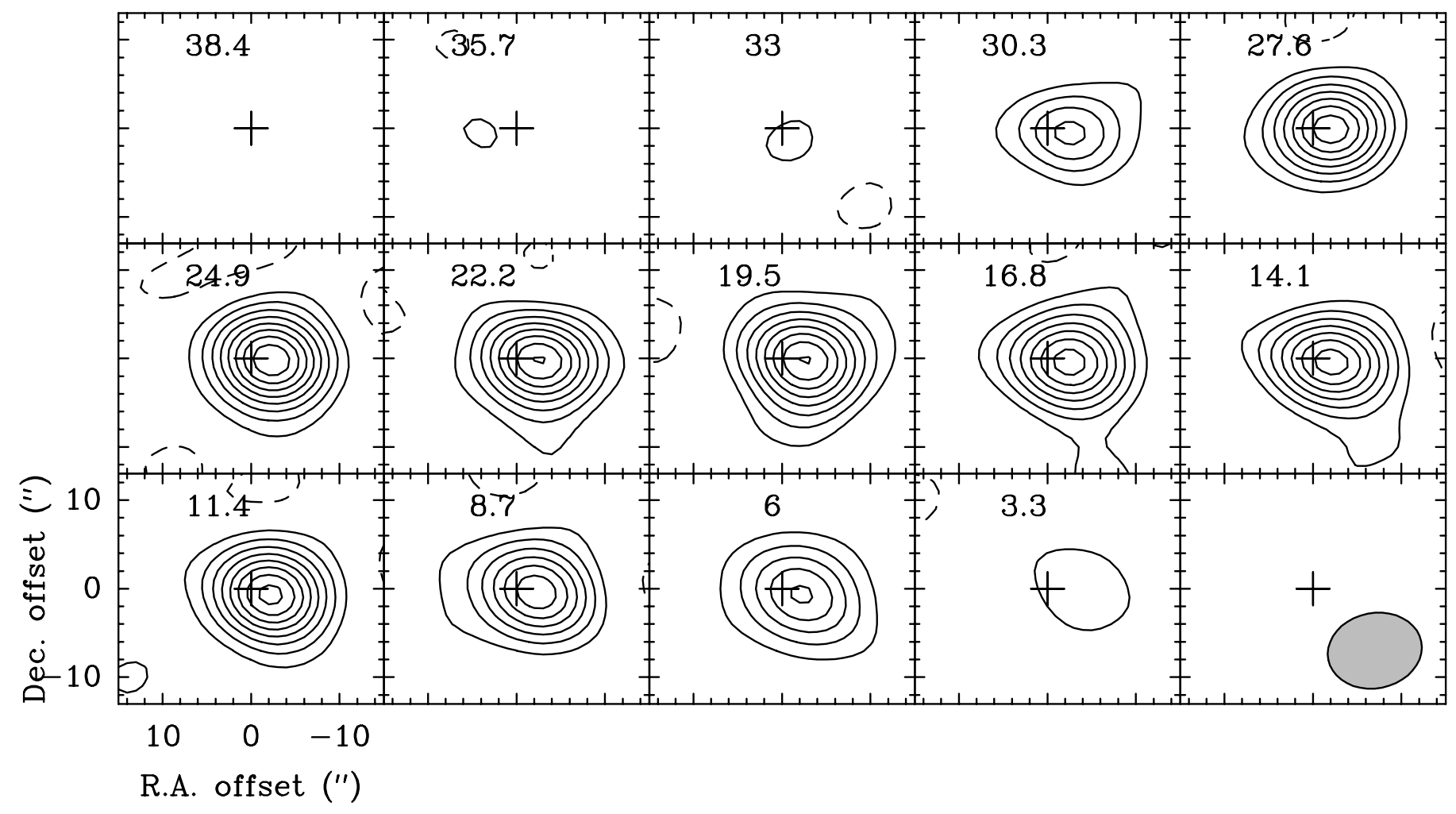

FIG. 7.- ${ }^{13} \mathrm{CO} J=1-0$ channel maps of IRAS $19475+3119$ obtained at OVRO. Each panel covers a velocity bin of width $2.7 \mathrm{~km} \mathrm{~s} \mathrm{~s}^{-1}$, centered at the LSR velocity shown near the top left corner. Contour levels run from $3 \sigma$ in $3 \sigma$ steps, where $\sigma=20 \mathrm{mJy} \mathrm{beam}^{-1}$ is the rms noise. As in Fig. 6 , the cross is located at the tracking center of the observations.

much larger than $20 \%$, the above-stated uncertainties are a reasonably good estimate of total errors in our flux measurement.

\section{DISTANCE, LUMINOSITY, AND THE CENTRAL STAR}

We adopt a distance of $4.9 \mathrm{kpc}$ to I19475, which corresponds to the far kinematic distance to I19475 (SC06) and is in accord with other recent studies of this object (e.g., SS06; Hrivnak \& Bieging 2005). At $4.9 \mathrm{kpc}, \mathrm{I} 19475$ has a total luminosity of $8300 \mathrm{~L}_{\odot}$.

The near-kinematic distance, estimated to be $1.7 \mathrm{kpc}$, using the systemic radial velocity, $V_{\mathrm{lsr}}=18 \mathrm{~km} \mathrm{~s}^{-1}$ and a simple Galactic rotation model ${ }^{11}$ gives a bolometric luminosity of $1000 L_{\odot}$, much too low for a PPN.

We have measured the fluxes of the central star, using the unsaturated, short-exposure images for each filter $(0.8$ and $0.2 \mathrm{~s}$ for F435W and F606W, respectively). We find that the central star has fluxes of $3.48 \times 10^{-13}$ and $4.22 \times 10^{-13} \mathrm{ergs} \mathrm{cm}^{-2} \mathrm{~s}^{-1} \AA^{-1}$ at 0.43 and $0.6 \mu \mathrm{m}$. The fluxes were measured using small apertures (with diameters of 8 and 10 pixels and aperture correction factors of 1.26 and 1.23 at 0.43 and $0.6 \mu \mathrm{m}$, respectively) to minimize the nebular contribution. From the above fluxes, we derive $B=10.7$, $V=9.66$, and $R=9.38$ (as in $\S 3.1$ ). Using the intrinsic $B-V$, i.e., $(B-V)_{0}=0.25$ for 119475 's central star (SS06), we find a total reddening toward the star of $E(B-V)=0.81$, corresponding to a total extinction toward the central star of $A_{V}=2.4$. We estimate the interstellar extinction to I19475 to be $A_{V}$ (ism) $=$ $0.70 \pm 0.49$, supplemented by an additive correction factor (to correct for a systematic extinction underestimation between 1 and $5 \mathrm{kpc}$ ) of 0.80 , giving a total $A_{V}(\mathrm{ism})=1.5 \pm 0.49$. These es-

11 I.e., $v_{r}=A D \sin 2 l \cos ^{2} b$ (Goodrich 1991), where $v_{r}$ is the radial velocity, $A=14.4 \pm 1.2 \mathrm{~km} \mathrm{~s}^{-1} \mathrm{kpc}^{-1}, D$ is the near-kinematic distance in kpc, and $(l, b)=\left(67.16^{\circ},-2.73^{\circ}\right)$ are the Galactic coordinates of $\mathrm{I} 19475$. timates have been made using a numerical algorithm that computes the three-dimensional visual interstellar extinction and its error from inputs of Galactic longitude and latitude, and distance, using a synthesis of several published studies (Hakkila et al. 1997). Hence the circumstellar extinction of the central star is $A_{V} \sim 0.9$, which is consistent with the value of $A_{V}$ derived for the model dust shell of I19475 by SS06.

Our value of the total extinction $A_{V}=2.4$ toward I19475 is higher than that derived by SS06 $\left(A_{V}=1.3\right)$ because they used the ground-based photometry of I19475, which includes contribution from the nebular light, and the nebular light is bluer than the observed starlight - the 0.43 to $0.6 \mu \mathrm{m}$ flux ratio for the nebula as a whole is 1.37 , which is significantly higher than that for the starlight, 0.82 . We have derived the nebular fluxes $\left(3.02 \times 10^{-13}\right.$ and $2.21 \times 10^{-13} \mathrm{ergs} \mathrm{cm}^{-2} \mathrm{~s}^{-1} \AA^{-1}$ at 0.43 and $0.6 \mu \mathrm{m}$, respectively) by subtracting the central star fluxes from the total (nebular+star) fluxes.

\section{LOBES AND HALO}

The color of the nebula is fairly uniform (Fig. 4). In the lobes, the color at any point $P$ is determined by three factors, namely, (1) reddening due to extinction of the starlight along the path from the star to point $P$, (2) reddening due to interstellar extinction, and (3) blueing due to scattering in the lobe wall at point $P$. The value of $B-V$ for the nebula as a whole is 0.47 , showing that it is redder than the intrinsic starlight, which has $(B-V)_{0}=$ 0.25 , but bluer than the observed color of the star, $B-V=1.0$. Measuring the colors of the lobes near their tips (which are least affected by the light from the halo component), we find lobes $\mathrm{d}$ and $\mathrm{d}^{\prime}$ are the bluest, with $B-V \approx 0.3$, whereas lobes a and $\mathrm{c}$ are less blue, with $B-V \approx 0.6$. Since lobe $\mathrm{b}$ is so faint, it is significantly contaminated by the halo light (the halo is redder than 


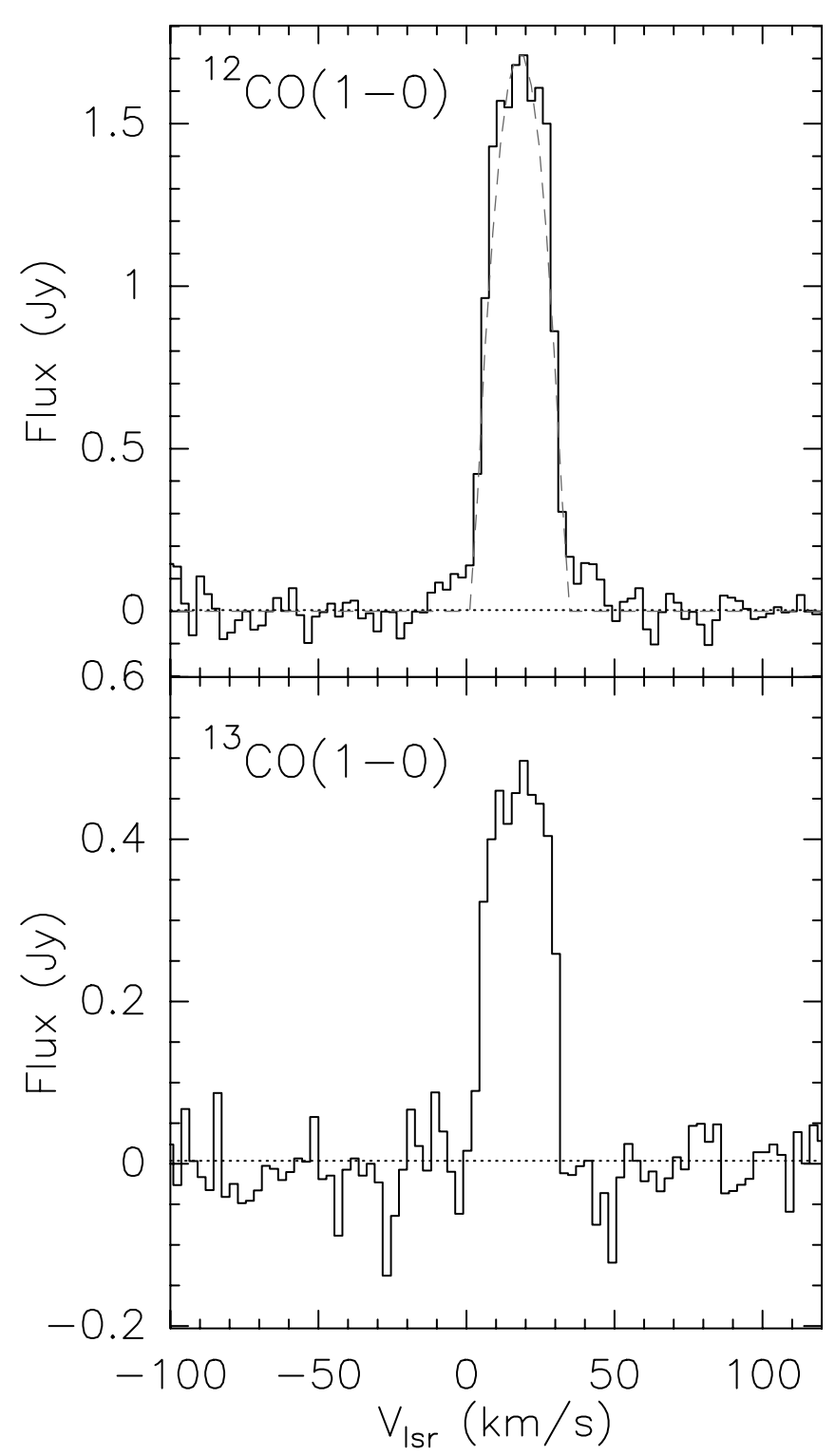

FIG. 8. $-{ }^{12} \mathrm{CO}$ and ${ }^{13} \mathrm{CO} J=1-0$ spectra of IRAS $19475+3119$ obtained at OVRO. The dashed curve in the top panel shows an empirical fit to the shape of the core emission.

lobe d, with $B-V \approx 0.6$ ), thus making it difficult to measure its color accurately. However, a measurement on the bright limb of this lobe gives $B-V \approx 0.4$, indicating that its real color is probably similar to that of lobe $d$. The bluer color of the lobe-pair $\mathrm{b}-\mathrm{d}$ compared to the $\mathrm{a}-\mathrm{c}$ pair, indicates that there is a larger amount of radial extinction from the central star to the scattering walls in the latter.

Some fraction of the halo light could result from the pointspread function (PSF) of the bright central source. In order to estimate this fraction, we compare the ratio of the surface brightness of the diffraction spikes relative to the surrounding nebulosity, $R$ (spk/halo), in a PSF image and the I19475 image at radial locations not affected by the presence of the lobes (radii $\sim 2^{\prime \prime}-3^{\prime \prime}$ ). We find that the value of $R$ (spk/halo) in the F435W (F606W) image of I19475 is a factor of 4-6 smaller than in the PSF image. We therefore conclude that the PSF of the bright central star in I19475 contributes only a small fraction of the observed brightness of the halo. The dominant fraction of halo light most likely represents scattered light from dust in the progenitor AGB cir- cumstellar envelope that has not yet been significantly affected by the processes that have formed the lobes. We have measured the radial profiles of the 0.43 and $0.6 \mu \mathrm{m}$ surface brightness using radial cuts generated from the F435W and F606W images (Fig. 10). In these cuts the surface brightness has been averaged over large angular ranges $\left(48^{\circ}\right.$ in the northwest and southeast quadrants) selected to avoid the presence of the diffraction spikes and the lobes, in order to increase the $\mathrm{S} / \mathrm{N}$ in the very faint outer regions. We find that the surface brightness, both at 0.43 and $0.6 \mu \mathrm{m}$, varies as $r^{-\alpha}$, with $\alpha \sim 4.1 \pm 0.2$, in the "halo" region $\left(r \gtrsim 1.2^{\prime \prime}\right)$ (Fig. 10). The halo can be seen out to a radius of about $6^{\prime \prime}$ or $4.4 \times 10^{17} \mathrm{~cm}(D / 4.9 \mathrm{kpc})$ in the $\mathrm{F} 435 \mathrm{~W}$ image, where it becomes limited by uncertainties in the background sky level. The size of the halo implies that the dense mass-loss phase of I19475's AGB progenitor lasted for at least $0.9 \times 10^{4} \mathrm{yr}$ $\left(15.0 \mathrm{~km} \mathrm{~s}^{-1} / V_{e, \text { agb }}\right)(D / 4.9 \mathrm{kpc})$. The observed value of $\alpha$ in the halo is inconsistent with an inverse-square radial density law (as, e.g., resulting from a constant mass loss at a constant expansion velocity). Assuming no significant change in the scattering properties of the dust or the outflow velocity with time, we conclude that the mass-loss rate increased roughly linearly with time in the past $10^{4} \mathrm{yr}$ of the mass-loss history of the AGB progenitor. Our timescale for this "heavy" mass-loss is significantly larger than that estimated by SC06 ( $\sim 4000$ yr; derived by appropriately scaling their estimate of the timescale, 5000-6000 yr for the higher value of the expansion velocity adopted by us). SC06 base their estimate on an outer radius of $2 \times 10^{17} \mathrm{~cm}$ for their model molecular shell.

\section{MOLECULAR GAS}

We now derive the physical parameters of the molecular shell producing the central emission component in the CO $J=1-0$ line-we do not further address the high-velocity outflow detected in our CO $J=1-0$ data in view of the detailed spatiokinematic modeling presented by SC06. In our modeling of the central emission component, we have used a somewhat different approach than that the one in SC06. We use an analytical expression for the brightness temperature of a spherical cloud with an $r^{-2}$ density law expanding at a constant speed, as described by Olofsson et al. (1990). This choice is dictated by the availability of the analytical expression for $\mathrm{CO}$ emission in the $r^{-2}$ case, which enables us to carry out a least-squares, multiparameter fit to our data and data on other CO lines available in the literature. Since our optical data indicate that the spherical shell has an $r^{-3}$ density law, we discuss later the uncertainty in our modeling introduced by adopting an $r^{-2}$ density. We note that the $J=1-0$ line is typically less optically thick than the $J=2-1$ line and is therefore potentially more sensitive to the total mass than the latter. The $\mathrm{CO}$ excitation is described by a fixed rotational temperature, $T_{\text {rot }}$, that is taken to be equal to the kinetic temperature, $T_{\text {kin }}$; i.e., we assume that the $\mathrm{CO}$ lines are thermalized. This is a reasonable approximation for most PPNe, which usually have densities larger than the $\operatorname{CO} J=1-0$ critical density $\left(\approx 10^{3} \mathrm{~cm}^{-3}\right)$ in most regions of their envelopes. In particular, for I19475, using SS06's value of the total shell mass, we find that the number density is about $2 \times 10^{3} \mathrm{~cm}^{-3}$ in the outermost regions of the shell, i.e., at a radius of about $2.5^{\prime \prime}$, and increases steeply inward. The $[\mathrm{CO}] /\left[\mathrm{H}_{2}\right]$ abundance ratio is assumed to be $4 \times 10^{-4}$ (as in SC06).

The fitting parameters of our model are the inner and outer radii ( $R_{\mathrm{in}}$ and $R_{\mathrm{ou}}$, respectively) of the expanding shell, the shell mass and the kinetic temperature. SC06 determine from their $\mathrm{CO}$ maps that the radius of the central cavity is about $1^{\prime \prime}$, and that the outer radius extends beyond $2.2^{\prime \prime}$. Based on our $J=1-0$ mapping, which shows the $J=1-0$ emission to be unresolved by the 


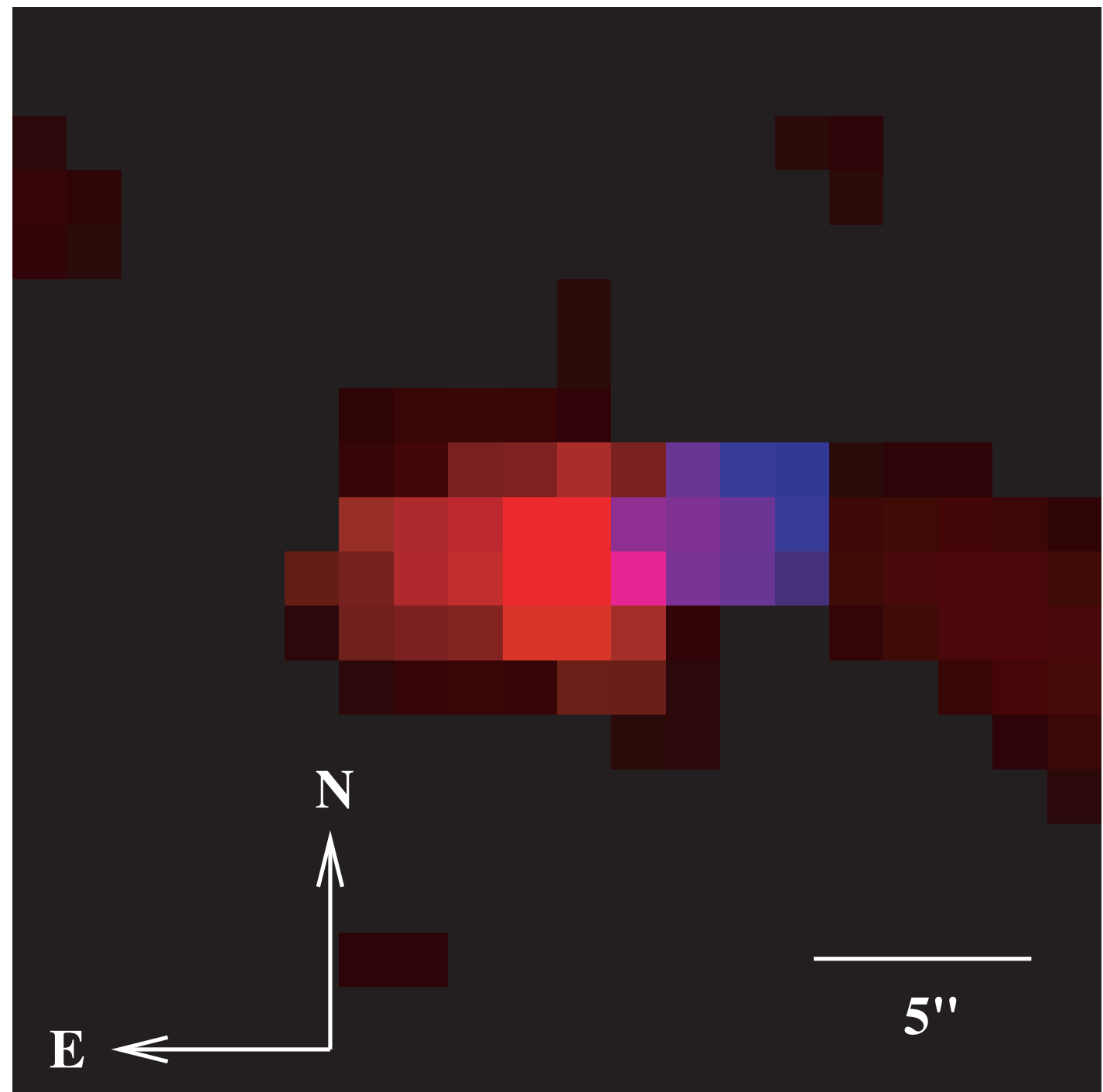

FIG. 9.- Map of the high-velocity CO $J=1-0$ emission of IRAS $19475+3119$ obtained at OVRO. The red (blue) emission represents the intensity integrated from $V_{\mathrm{lsr}} 34.9$ to $47.9 \mathrm{~km} \mathrm{~s}^{-1}\left(-14.5\right.$ to $\left.1.1 \mathrm{~km} \mathrm{~s}^{-1}\right)$.

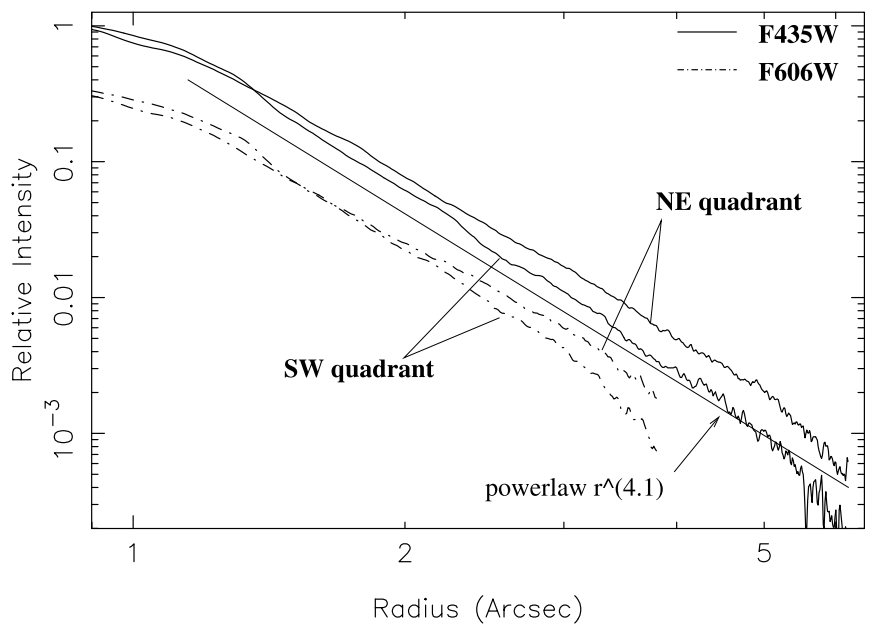

FIG. 10. - Radial profiles of halo surface brightness at 0.43 (solid curves) and $0.6 \mu \mathrm{m}$ (dash-dotted curves) generated from the $\mathrm{F} 435 \mathrm{~W}$ and $\mathrm{F} 606 \mathrm{~W}$ images. The surface brightness has been averaged over large angular ranges, which avoid the nebular lobes $\left(48^{\circ}\right.$ in the northeast and southwest quadrants) in order to increase the $\mathrm{S} / \mathrm{N}$ in the very faint outer regions of the halo. A radial power law, with exponent -4.1 , is shown for comparison. [See the electronic edition of the Journal for a color version of this figure.] $9.2^{\prime \prime} \times 6.7^{\prime \prime}$ beam, they argue that the outer radius cannot be much greater than about $2.75^{\prime \prime}$ from considerations, explained in their $\S 4.2 .1$ and summarized as follows. From their high angular resolution $J=2-1$ observations, they derive a size for the halo of about $4.4^{\prime \prime} \times 4.4^{\prime \prime}$, i.e., $R_{\mathrm{ou}} \approx 2.2^{\prime \prime}$ (these numbers were obtained by deconvolving the beam from the data and are therefore smaller than the size of the map in their Fig. 3). Since part of the $J=2-1$ emission from the extended halo is filtered out from the interferometer, SC06 infer that $R_{\mathrm{ou}}$ is probably larger than $2.2^{\prime \prime}$. However, it cannot be much larger than $2.75^{\prime \prime}$ because in that case it would start to get spatially resolved in the $J=1-0$ maps presented in this paper. We therefore set the search range for $R_{\text {in }}$ to be within $\pm 20 \%$ of $1^{\prime \prime}\left(\right.$ or $\left.7.4 \times 10^{16} \mathrm{~cm}\right)$, and $R_{\text {ou }}$ to lie in the range $2.2^{\prime \prime}-2.8^{\prime \prime}$, or $(1.6-2.0) \times 10^{17} \mathrm{~cm}$.

We have fitted the fluxes of the ${ }^{12} \mathrm{CO} J=1-0$ and $2-1$ lines observed at OVRO, as well as previously published single-dish data (see Table 1). The latter include: (1) IRAM $30 \mathrm{~m}$ observations of the $J=1-0$ and 2-1 lines and (2) Mt. Graham $12 \mathrm{~m}$ observations of the $J=2-1$ and 4-3 lines. Our least-squares minimization procedure finds reasonable fits to all the data with $R_{\text {in }}$ and $R_{\text {ou }}$ in the above-defined ranges, $T_{\text {rot }}=14.5-21 \mathrm{~K}$, and a shell mass $\gtrsim 0.4 M_{\odot}$. The lower limit on the shell mass is due to 
TABLE 1

Observational and Model Results

\begin{tabular}{|c|c|c|c|c|c|c|}
\hline Line & Dish/HPBW & $\begin{array}{c}\text { Peak }^{\mathrm{a}} \\
(\mathrm{K} \text { or Jy) }\end{array}$ & $\begin{array}{c}\text { Model } \\
\text { (K or Jy) }\end{array}$ & $\begin{array}{c}\text { Area } \\
\left(\mathrm{Jy} \mathrm{km} \mathrm{s}^{-1}\right)\end{array}$ & $\begin{array}{c}\text { Model } \\
\left(\mathrm{Jy} \mathrm{km} \mathrm{s}^{-1}\right)\end{array}$ & References \\
\hline $1-0 \ldots \ldots$ & OVRO & 1.68 & 1.74 & 42.9 & 39.6 & 1 \\
\hline 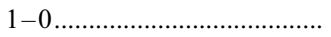 & IRAM $30 \mathrm{~m} / 22^{\prime \prime}$ & 0.37 & 0.32 & $\ldots$ & $\ldots$ & 2 \\
\hline $2-1$ & IRAM $30 \mathrm{~m} / 12.5^{\prime \prime}$ & 0.75 & 0.89 & 126 & 144 & 3 \\
\hline 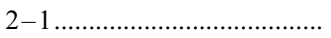 & HНT $10 \mathrm{~m} / 32^{\prime \prime}$ & 0.17 & 0.14 & $4.1^{\mathrm{b}}$ & $3.2^{\mathrm{b}}$ & 4 \\
\hline $4-3$ & HHT $10 \mathrm{~m} / 17^{\prime \prime}$ & 0.37 & 0.35 & $11^{\mathrm{b}}$ & $7.8^{\mathrm{b}}$ & 4 \\
\hline 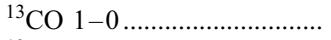 & OVRO & 0.45 & 0.47 & 11.3 & 13.0 & 1 \\
\hline${ }^{13} \mathrm{CO} 1-0$ & IRAM $30 \mathrm{~m} / 23^{\prime \prime}$ & 0.11 & 0.09 & $\ldots$ & $\ldots$ & 2 \\
\hline
\end{tabular}

${ }^{\text {a }}$ Peak: peak $T_{\mathrm{mb}}$ for single-dish observations; peak spatially integrated flux for OVRO interferometric data.

b In units of $\mathrm{K} \mathrm{km} \mathrm{s}^{-1}$ from Hrivnak \& Bieging (2005); both observed values include the contribution from the high-velocity wings, which is not included in the model.

References.-(1) This paper; (2) Sánchez Contreras et al. 2006; (3) Bujarrabal et al. 2001; (4) Hrivnak \& Bieging 2005.

all of the $\mathrm{CO}$ lines being optically thick; e.g., the smallest tangential optical depth of the CO $J=1-0$ line in our models at half the outer radius of the shell is 4.8 (for reference, the corresponding optical depths of the 2-1 and 4-3 lines are 12.2 and 7.8, respectively). The mass-loss rate, $\dot{M} \gtrsim 2 \times 10^{-4} M_{\odot} \mathrm{yr}^{-1}$, and the corresponding mass-ejection timescale, given by $\left(R_{\mathrm{ou}}-R_{\mathrm{in}}\right) / V_{e, \mathrm{AGB}}$, is about 2000 yr. SC06's derived value of the temperature and shell mass $\left(14 \mathrm{~K}\right.$ and $\left.0.4 M_{\odot}\right)$ are consistent with our ranges for these parameters; their value of $\dot{M}$ is $\sim 10^{-4} M_{\odot} \mathrm{yr}^{-1}$, not significantly different from our value. ${ }^{12}$ SC06 have explored constant density as well as $r^{-3}$ density-law models, and do not find significant differences in their results for the shell mass and temperature. The good agreement of our results with theirs indicates that the derived values of the temperature and mass are not very sensitive to the density law. The observational data which we have fitted and the corresponding model values are tabulated below for our lowest shell-mass model.

\subsection{The ${ }^{13} \mathrm{C}$ to ${ }^{12} \mathrm{C}$ Isotope Ratio}

Since we can only estimate a lower limit to optical depth of the ${ }^{12} \mathrm{CO} J=1-0$ line from our CO modeling, we can only derive a upper limit to the ${ }^{13} \mathrm{C}$ to ${ }^{12} \mathrm{C}$ abundance ratio, $f_{13 / 12}$, in I19475 from fitting our ${ }^{13} \mathrm{CO} J=1-0$ OVRO flux as well as the peak line intensity observed with the IRAM $30 \mathrm{~m}$ dish. Using our lowest shell-mass model, we find $f_{13 / 12} \lesssim 0.1$. In this model the ${ }^{13} \mathrm{CO}$ line has a modest tangential optical depth of about 0.45 at half the outer radius of the shell. Only a model which selfconsistently computes the kinetic temperature (from a detailed consideration of gas heating and cooling mechanisms), the CO emission, and the gas-to-dust ratio, as described in Sahai (1990) for AGB circumstellar envelopes, can provide an accurate estimate of the ${ }^{12} \mathrm{CO}$ optical depth and thus the ${ }^{13} \mathrm{C}$ to ${ }^{12} \mathrm{C}$ abundance ratio in this object. Although such modeling is outside the scope of this paper, it is quite important because the $f_{13 / 12}$ ratio is a probe of the main-sequence mass of the central star, which is very difficult to determine otherwise. High values of $f_{13 / 12}$ (i.e., $\gtrsim 1 / 15$ ) require hot-bottom-burning (HBB) nucleosynthesis, a process that only operates for stars whose main-sequence masses are larger than about 3.5-4 $M_{\odot}$ (see, e.g., the detailed discussion in Sahai et al. 2006).

\section{DUST}

By modeling the SED of I19475 from the optical to the farinfrared using a one-dimensional dust radiative transfer code,

\footnotetext{
${ }^{12}$ Part of the discrepancy is due to their values of the expansion velocity $\left(11 \mathrm{~km} \mathrm{~s}^{-1}\right)$ and distance $(4.8 \mathrm{kpc})$ being less than ours.
}

SS06 derive a mass of $\gtrsim 1 M_{\odot}\left[34 \mathrm{~cm}^{2} \mathrm{~g}^{-1} / \kappa(100 \mu \mathrm{m})\right](\delta / 200)$, where $\kappa(100 \mu \mathrm{m})$ is the $100 \mu \mathrm{m}$ dust mass absorption coefficient (per unit dust mass) and $\delta$ is the gas-to-dust ratio. The inner and outer radii of the shell are $0.88 \times 10^{17}$ and $4.4 \times 10^{17} \mathrm{~cm}$, and dust temperatures ranging from about 94 to $46 \mathrm{~K}$ provide the best fit. In agreement with our results from the HST images for the halo, this model supports an $r^{-3}$ density law for the dust shell.

The values of $R_{\text {in }}$ derived from the dust model and our $\mathrm{CO}$ model are quite comparable. The difference between the values of $R_{\mathrm{ou}}$ for the dust shell and the CO shell may be real or may result from the uncertainties in the $\mathrm{CO}$ and dust modeling. The former is constrained by fitting the full SED using a spherical shell, but since the SED includes some contribution from dust emission in the walls of the lobes seen in our HST images, the inner and outer radii of the shell used to approximate emitting region is somewhat imprecise. In any case, our imaging directly shows that the outer radius of the dust shell is larger than $3.7 \times$ $10^{17} \mathrm{~cm}(\S 5)$, i.e., larger than the outer radius of the CO shell. In our CO modeling, if we were to incorporate an $r^{-3}$ density law, larger values of $R_{\text {ou }}$ (which was constrained by the requirement that the $\operatorname{CO} J=1-0$ emission be unresolved by our interferometric observations) would be admissible, since due to the more rapid decrease of the density with radius, the emission from the outer regions of such a shell would be less than that from the assumed $r^{-2}$ shell. Furthermore, if one took into account a realistic radial temperature gradient in the shell (typically, $T_{\text {kin }} \sim r^{-\beta}$, where $\beta \sim 1$; e.g., Sahai 1990), the outermost regions could have significantly lower excitation, producing a rapidly decreasing contribution to the $\mathrm{CO}$ emission with radius. As a result of the above two effects, the CO-emitting region would appear smaller than the real physical size of the shell. We conclude that the $\mathrm{CO}$ data does not probe the full mass of the shell out to the outer radius inferred from the dust modeling. Hence, the shell mass derived above from our $\mathrm{CO}$ data is quite consistent with the total circumstellar mass as estimated from the far-infrared dust emission of this source. If we use the SS06 model density to compute the total mass out to the outer radius of the $\mathrm{CO}$ shell in our lowest mass CO model, we find a value of about $0.5 M_{\odot}$.

\subsection{The Millimeter/Submillimeter Excess and Large Grains}

SS06 estimated that the source flux at submillimeter wavelengths $(850 \mu \mathrm{m})$ is $\gtrsim 20 \mathrm{mJy}$, using the flux measured by Gledhill et al. (2002) and roughly correcting for the contribution of the $\mathrm{CO}$ $J=3-2$ line. Since the corrected $850 \mu \mathrm{m}$ flux was a factor $\gtrsim 2$ larger than the model flux, SS06 suggested that the former most likely arises from large, cool dust grains. We have used an estimate of the line flux based on our modeling described above and 
the response curve of the $850 \mathrm{~N}$ SCUBA filter used for the $850 \mu \mathrm{m}$ continuum observations, and we find that the $850 \mu \mathrm{m}$ flux is about $24 \mathrm{mJy}$. Our detection of the $2.6 \mathrm{~mm}$ continuum supports this result - the observed flux is a factor 20 larger than the model flux of SS06.

Since the presence of $\mathrm{H} \alpha$ emission suggests that a compact region of ionized gas surrounds the central star, we examine whether the free-free continuum emission from this region can account for the (corrected) $850 \mu \mathrm{m}$ and $2.6 \mathrm{~mm}$ fluxes. We find that the power-law fit $\left(F_{\nu} \propto \nu^{-p}\right)$ to these two points gives an exponent $p \sim 1.8$, implying that the emission cannot arise from optically thin free-free emission, for which $p \sim 0$. If we assume that the emission is produced by optically thick gas with optical depth $\tau$ at $850 \mu \mathrm{m}$, then from the total observed $\mathrm{H} \alpha$ emission-line flux $\left(\sim 6 \times 10^{-14} \mathrm{ergs} \mathrm{cm}^{-2} \mathrm{~s}^{-1}\right)$ and given an electron temperature of $10^{4} \mathrm{~K}$, we find that the free-free emission at $850 \mu \mathrm{m}$ and $2.6 \mathrm{~mm}$, for, say, $\tau=1$, is 0.02 and $0.004 \mathrm{mJy}$, far too low to explain the observed submillimeter and millimeter fluxes. ${ }^{13}$

We therefore attribute the emission at these wavelengths to thermal dust emission. As an additional check, we have examined archival radio continuum data on I19475, which can provide constraints on the size of any region of optically thick ionized gas in I19475. Observations at the position of I19475 were made with the NRAO Very Large Array (VLA) ${ }^{14}$ during the NRAO VLA Sky Survey in the D configuration (NVSS; Condon et al. 1998) at $20 \mathrm{~cm}$ on 1995 April 28 with an angular resolution of $45^{\prime \prime}$. No radio emission was detected; the $1 \sigma \mathrm{rms}$ noise was $0.4 \mathrm{mJy}^{\text {beam }}{ }^{-1}$. Further VLA observations at a wavelength of $6 \mathrm{~cm}$ were found in the NRAO archive, made using the A configuration on 1987 September 19 , with an angular resolution of approximately $0.5^{\prime \prime}$. No radio emission was detected at $6 \mathrm{~cm}$; the rms noise was $70 \mu \mathrm{Jy} \mathrm{beam}^{-1}$. Assuming an electron temperature of $10^{4} \mathrm{~K}$, the diameter of the ionized gas region must be less than 20 mas at $6 \mathrm{~cm}$ and 170 mas at $20 \mathrm{~cm}$ (the $20 \mathrm{~cm}$ limit reflects the higher noise level and longer wavelength of these observations compared to the $6 \mathrm{~cm}$ observations). Thus, the physical diameter of any region of optically thick ionized gas, if present, must be less than $1.6 \times 10^{15} \mathrm{~cm}$.

The ratio of the $850 \mu \mathrm{m}$ to the $2.6 \mathrm{~mm}$ flux is about $6-7$, similar to the ratio for blackbody emission at 20-30 K, suggesting that the dust grains responsible for producing the millimeter-and submillimeter-wave emission are about as large as $2.6 \mathrm{~mm}$. We have revised the SS06 best-fit model (number 7 in their Table 5) to include a large-grain-component shell and have varied its major characteristics: the dust temperature $T_{d}$, the optical depth at $100 \mu \mathrm{m}, \tau(100)$, and the grain size parameter $a$. The ratio of the outer to inner radius is arbitrarily assumed to be close to unity (1.1). We find that with $T_{d} \sim 30 \mathrm{~K}$ and $a \sim 2.6 \mathrm{~mm}$, about $0.043 M_{\odot}$ of dust is required to produce the $850 \mu \mathrm{m}$ flux (Fig. 11). We have assumed the dust mass extinction coefficient $\kappa$, at $\lambda \ll a$ (say at $100 \mu \mathrm{m}$ ), to be $1 \mathrm{~cm}^{2} \mathrm{~g}^{-1}$, derived from $\kappa=\sigma\left(4 \pi a^{3} \rho_{d} / 3\right)^{-1}$, with $\sigma=\pi a^{2}, a=2.6 \mathrm{~mm}$, and $\rho_{d}=3 \mathrm{~g} \mathrm{~cm}^{-3}$. The large grains must be located at a distance of about $1.3 \times 10^{17} \mathrm{~cm}$, or $1.7^{\prime \prime}$, from the central star. High-sensitivity interferometric mapping at $850 \mu \mathrm{m}$ is needed to pin down the location of these grains. The model $2.6 \mathrm{~mm}$ flux is $1.8 \mathrm{mJy}$, somewhat lower than our observed value, and the $1.3 \mathrm{~mm}$ flux is $11 \mathrm{mJy}$, consistent with the upper limit of $12 \mathrm{mJy}$ found by SC06.

Although the $2.6 \mathrm{~mm}$ model flux is still less than observed, given the inherently large uncertainties in the size distribution

\footnotetext{
13 See, e.g., Jura et al. (2001) for a similar calculation in the case of SS Lep.

14 The National Radio Astronomy Observatory is a facility of the National Science Foundation, operated under cooperative agreement by Associated Universities, Inc.
}

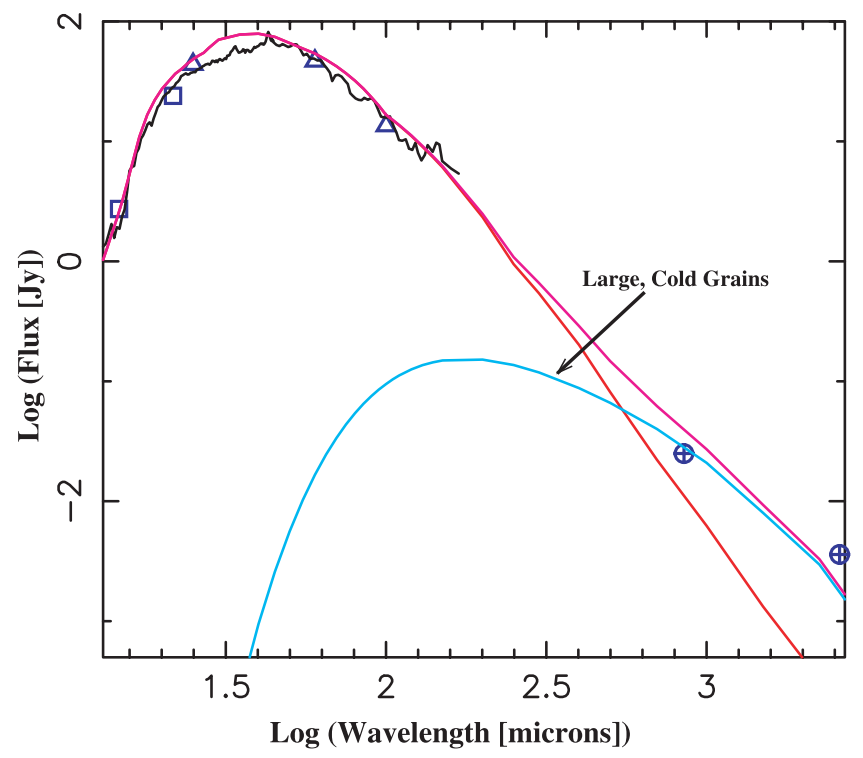

FIG. 11.-Observations (black curve: ISO spectra; blue symbols: photometric data (circled crosses: SCUBA on JCMT $0.88 \mathrm{~mm}$ and OVRO $2.6 \mathrm{~mm}$; squares: MSX; triangles: IRAS; and magenta curve: model spectrum) of I 19475. Individual components of the model are also shown: warm (red) and cold (cyan) shells. The warm shell represents the best fit model from Sarkar \& Sahai (2006) derived to fit the observed SED out to $\sim 200 \mu \mathrm{m}$, and the cold shell represents the large-grain emission component derived to fit submillimeter and millimeter continuum data.

and emission/scattering properties of the large grains, we have not attempted to further fine-tune our model for a better fit. We now examine in some detail the uncertainties in the absorption/ emission properties of large grains and their impact on our mass estimate. Jura et al. (2001) derive the mass in large $(a>0.2 \mathrm{~mm})$ grains around the red giant SS Lep from its $850 \mu \mathrm{m}$ flux, using $M_{\text {dust }}=\left(F_{\nu} D^{2}\right) /\left[B_{\nu}\left(T_{d}\right) \chi_{\nu}\right]$ and adopting the Rayleigh-Jeans approximation to the blackbody function. These authors adopt $\chi_{\nu}(850 \mu \mathrm{m}) \sim 3 \mathrm{~cm}^{2} \mathrm{~g}^{-1}$, referring to an extensive study by Pollack et al. (1994) on the composition and radiative properties of dust grains. Using this study, we find lower values of $\chi_{\nu}(850 \mu \mathrm{m})$ for millimeter-sized grains compared to Jura et al.'s value. In their Table 4, Pollack et al. list $\kappa_{t}$, the absorption coefficient per unit total mass (i.e., of gas and dust) ${ }^{15}$ at $\lambda=1 \mathrm{~mm}$ for grains of different sizes, as well as $\beta$, the spectral index of the absorption coefficient for $\lambda=650-2700 \mu \mathrm{m} .{ }^{16}$ We first derive conservative dust masses by using the largest values of $\kappa_{t}$ given by Pollack et al. (1994) for low-temperature (100 K), large grains. Thus, for grains with $a=300 \mu \mathrm{m}$, for which $\kappa_{t}=0.014 \mathrm{~cm}^{2} \mathrm{~g}^{-1}$ and $\beta=1.81$, we get $\chi_{\nu}(850 \mu \mathrm{m})=g_{d(P)}\left[0.014(1 / 0.85)^{1.81}\right]=1.34 \mathrm{~cm}^{2} \mathrm{~g}^{-1}$. Similarly, for grains with $a=3000 \mu \mathrm{m}$, we get $\chi_{\nu}(850 \mu \mathrm{m})=$ $g_{d(P)}\left[7.4 \times 10^{-3}(1 / 0.85)^{1.34}\right]=0.66 \mathrm{~cm}^{2} \mathrm{~g}^{-1}$. Interpolating between the above values of $\chi_{\nu}(850 \mu \mathrm{m})$ for $a=300$ and $3000 \mu \mathrm{m}$, we estimate $\chi_{\nu}(850 \mu \mathrm{m})=0.69 \mathrm{~cm}^{2} \mathrm{~g}^{-1}$ for $a=2.6 \mathrm{~mm}$ grains; using this value and $F_{\nu}(850 \mu \mathrm{m})=24 \mathrm{mJy}$, we get $M_{\text {dust }}=$ $0.047 M_{\odot}$. Other varieties of grains with $a=300$ and $3000 \mu \mathrm{m}$ in Table 4 of Pollack et al. have $\kappa_{t}$ values that are lower than the

15 However, we are interested in the dust cross-section per unit dust masshence, we scale Pollack et al.'s value by the factor $g_{d(P)}=71.4$, where $g_{d(P)}$ is the gas-to-dust mass ratio derived from their Table 2 . The dust cross-section per unit dust mass is of course independent of the gas-to-dust ratio and so can be applied to any environment to compute the dust mass, irrespective of the prevailing gasto-dust ratio in that environment.

16 We note that a common assumption about grains - that the spectral index is 0 if $a>\lambda /(2 \pi)$ - is not validated by the detailed study of Pollack et al. (1994); e.g., for grains with $a=3000 \mu \mathrm{m}$, the spectral index $\beta$ at $\lambda=650-2700 \mu \mathrm{m}$ for $100 \mathrm{~K}$ grains is found to lie in the range $\sim 1.1-1.4$. 
maximum value by a factor $\sim 1.5-2.5$; adopting these values will thus lead to correspondingly higher large-grain dust masses.

The emissivity could be larger than our assumed value if the dust particles consisted of large fluffy (i.e., porous) aggregates, resulting from, e.g., dust coagulation and ice accretion. Ossenkopf (1991) finds that grains with "medium internal densities," i.e., vacuum volume fractions $\sim 0.5$, can have mass absorption coefficients that are significantly increased over those of solid grains; e.g., in Figure 9 of Ossenkopf (1991) the increase amounts to a factor $\sim 2.2$ at the longest wavelengths shown, 100-200 $\mu \mathrm{m}$. Such an enhanced emissivity would lead to correspondingly lower largegrain dust masses.

In summary, in spite of the uncertainties in grain properties, it is clear that the mass of the large-grain component is quite substantial. If we assume a gas/dust ratio of $g_{d}=200$ in this component, as for the extended cool dust shell, the total mass associated with the large dust grains, $M_{\mathrm{lg}}$, could be as large as about $9 M_{\odot}$, which is disturbingly large for a PPN, given the widely held view that stars with progenitor masses $>8 M_{\odot}$ evolve into supernovae. Of course, it is quite possible that the gas-to-dust ratio in the region of large grains is lower than the typical value, as has been proposed for the large-grain region in the bipolar PPN IRAS $22036+5306$ (Sahai et al. 2006). If we assume $g_{d}=100$, typical of the interstellar medium, then $M_{\mathrm{lg}}=4.5 M_{\odot}$. Significantly lower gas-to-dust ratios have been derived by Sopka et al. (1985) from $400 \mu \mathrm{m}$ observations in the PPNs OH231.8+4.2 and AFGL 2688 (ratios of 14 and 31, respectively). So we if assume that $g_{d}$ in I19475 is lower by a factor 5-10 than the typical circumstellar value, i.e., $20-40$, we obtain a total mass of $0.86-1.7 M_{\odot}$ associated with the large-grain component, which, combined with the $1 M_{\odot}$ of material in the cool dust shell and the mass of the postAGB star (typically $0.6 M_{\odot}$ ), suggests that the main-sequence progenitor mass was at least $2.5 M_{\odot}$.

\section{THE STRUCTURE AND FORMATION OF I19475}

The detailed morphology of I19475 revealed by our data, as well as other preplanetary nebulae imaged in the past, has important implications for current models for the evolution of lowand intermediate-mass stars as they evolve from AGB red giants to planetary nebulae. There is now overwhelming observational evidence indicating that this evolution is accompanied by the onset of a fast wind (or winds) during the very late AGB and/or the early post-AGB phase. The wind(s) is (are) intrinsically collimated (Sahai 2002), not radiatively driven (Bujarrabal et al. 2001), and in strong interaction with the ambient circumstellar medium to produce elongated, evacuated lobe structures (Sahai 2002). Based on their imaging survey of young PNs, Sahai \& Trauger (1998) hypothesized that the collimated fast winds are the primary mechanism for the dramatic change in circumstellar geometry and kinematics as stars evolve off the AGB. Multipolar and/or point-symmetric morphologies, which are commonly seen in young PNs and PPNs, presumably then result from changes in the direction of the collimated fast winds.

We note that the quadrupolar morphology of I19475 is not a very common one among PPNs or PNs. This morphological class was first identified by Manchado et al. (1996), with five member PNs, although only two of these five (K3-24 and M2-46) display the quadrupolarity sufficiently clearly, such that two distinct bipolar lobes oriented along different symmetry axes can be obviously identified. In our extensive imaging surveys of PPNs with $H S T$, I19475 is the only example of a quadrupolar object.

The morphology of I19475, as revealed in our HST images, provides several clues to its formation. Our analysis of the halo (together with the $\mathrm{CO}$ and dust modeling) indicates that this was produced by the AGB progenitor ejecting mass in a spherically symmetric wind at a rate that increased linearly with time over a period of at least $10^{4} \mathrm{yr}$, reaching a value as high as few times $10^{-4} M_{\odot} \mathrm{yr}^{-1}$ near the end of this phase. Based on our estimates of the inner radius of the halo $\left(\sim 8 \times 10^{16} \mathrm{~cm}\right)$, this phase came to an end about $1700 \mathrm{yr}$ ago. For the formation of the quadrupolar structure, one can imagine two classes of models. In the first class, the jet-driven model, there are two possibilities. First, if we assume that the material in each of the two pairs of lobes has similar expansion velocities, then the significantly longer extent of the $\mathrm{b}-\mathrm{d}$ lobe pair compared to the $\mathrm{a}-\mathrm{c}$ pair implies that the former is older. In this case, we can imagine a scenario in which a bipolar jet first carved out the $b-d$ lobe pair, then switched its orientation by a large angle (about $35^{\circ}$ ) rather abruptly (i.e., on a timescale, $t_{\text {switch }}$, that is small compared to the lobe expansion timescales) to the position angle of the a-c lobes, and then proceeded to carve these out. The strikingly point-symmetric shape of the a-c lobe pair, in addition to the structure within the $b-d$ pair, suggests that the jet must have also changed its direction within a small solid angle about the average orientation, while generating each of these pairs of lobes, on a timescale significantly longer than $t_{\text {switch }}$. Surprisingly, however, the fast outflow seen in $\mathrm{CO}$ appears to be directed along the $b-d$ pair; its kinematical age is estimated to be $\sim 1900$ yr (SC06), implying that it started just as the AGB heavy mass-loss phase came to an end.

Alternatively, a pair of bipolar jets operate quasi-simultaneously with different orientations to produce the quadrupolar morphology. Such a pair of bipolar jets could result if (1) a rapidly rotating central object (disk or star) launched a wind that was collimated via magnetic hoop stresses; misalignment of the disk and star rotation axis would then produce quadrupolar lobes (see panel a.3 of Fig. 2 in Balick \& Frank 2002; also Blackman et al. 2001); or (2) the jets were driven from a pair of disks, either around a binary companion to the AGB star (as in Mastrodemos \& Morris 1998) plus around the progenitor AGB star itself, or around two non-AGB stars in a triple system.

A second class of model, proposed by Matt et al. (2004), invokes explosive, magnetically driven ejections from the (single) primary star occurring simultaneously along different directions. These authors show that in their model, mass can be simultaneously ejected at high speeds along a polar axis, as well as along the equatorial plane. Whether or not such a model can be modified to produce the quadrupolar structure found in I19475 remains to be seen.

No fast-moving gas can be identified in the $\mathrm{CO}$ data associated with the $\mathrm{a}-\mathrm{c}$ lobe pair; however, a pair of bright clumps, which are oriented along this lobe pair, can be seen in the $\mathrm{CO} J=2-1$ maps (SC06). It is possible that these clumps are, in fact, the result of shock compression resulting from the interaction of a fast bipolar outflow along the $\mathrm{a}-\mathrm{c}$ axis with the AGB ejecta, but the emission from fast-moving gas in the bipolar outflow itself is below the detection limit of the $\mathrm{CO}$ observations.

On the basis of their claim to have discovered a two-arm spiral structure in I19475, Gledhill et al. (2001) discuss the possibility that this results from the presence of a binary companion producing spiral-shaped accretion wakes in the AGB wind of the primary star, as seen in numerical simulations by Mastrodemos \& Morris (1999). But we do not see this spiral structure in our HST images, so its existence is open to question. Moreover, assuming that it is real, we find the comparison poorly supported by their data. First, at a distance of $4.9 \mathrm{kpc}$, the separation between the spiral peaks in their image would be a factor 250 times 
larger than the size of the region showing spiral structures in the simulations. ${ }^{17}$ Second, Gledhill et al. (2001) also point out that the simulations predominantly show a single spiral winding around the primary, in strong contrast to the two-armed spiral seen in the $J$-band image.

I19475 shows no presence of a dusty toroidal waist structure in the HST images, as is commonly seen in bipolar PPNs and the multipolar PPN IRAS 19024+0044. It is, of course, possible that such a dense toroid is present, forming a waist for the $b-d$ lobe pair, but cannot be seen due to the a-c lobes. Since the central star is seen directly in our images, such a torus is most likely not oriented edge-on and would appear as a bright elliptical structure in the absence of the a-c lobe-pair. However, the a-c lobe pair provides a screen with (1) significant optical depth (note that the circumstellar $A_{V}$ is about 0.9 toward the central star, most of which could arise in the a-c lobe pair) and (2) structured brightness against which the light from the torus may be difficult to delineate.

The presence of large (millimeter sized) grains in I19475 adds to a growing body of evidence that such grains may be common in PPNs. In recent papers Sahai et al. (2006) report the presence of such grains in the PPN IRAS 22036+5306, and they list other PPNs with large grains that have been reported in the literature (Red Rectangle; AFGL 2688), and Sánchez Contreras et al. (2007) find large grains in IRAS 18276-1431, a bipolar PPN very similar to AFGL 2688. It is interesting to note that except for I19475, all other objects show dense dusty waists, and at least in the case of IRAS $22036+5306$, the interferometric observations show the submillimeter emission of the large grains to be confined to, and therefore most likely associated with, the waist region. Thus, whereas in objects such as IRAS $22036+5306$ one may imagine a scenario in which the waists may provide the long timescales and high densities required for the grains to grow to millimeter sizes, the same does not apply to I19475 - unless it possesses a hidden waist. If the large grains are associated with one or both of the two lobe pairs, then at some stage in I19475's past the material in these lobes was probably confined to a more compact region with high densities, which allowed the grains to grow to large sizes.

\section{CONCLUSIONS}

Using HST's ACS instrument, we have obtained optical images of I19475 as part of our surveys of preplanetary nebulae. Optical spectroscopy and millimeter-wave (interferometric) mapping of its molecular line emission has been carried out using the Keck Observatory and the Owens Valley Radio Observatory, respectively. We find that:

1. I19475 is a quadrupolar nebula of size $\sim 10.5^{\prime \prime} \times 4.7^{\prime \prime}$ with two prominent, limb-brightened, elongated bipolar lobes emanating from the center of the nebula. The projected axes of these lobes are not orthogonal.

2. A very faint, round, diffuse halo surrounds the lobes. This halo has a power-law radial surface brightness profile with a power-law exponent of about -4 , and it most likely represents

\footnotetext{
17 Gledhill et al. (2001) noted this problem for a distance to I19475 as small as $1 \mathrm{kpc}$.
}

the remnant spherical circumstellar shell formed as a result of mass loss during the AGB phase, which has increased linearly with time over the past $10^{4} \mathrm{yr}$.

3. The optical spectroscopy reveals a complex $\mathrm{H} \alpha$ profile with a broad photospheric absorption feature, and a narrow inverse P Cygni shaped core. Comparison with previous multiepoch observations, in which the core consists of a double-peaked emission feature, suggests that our observed line probably represents the variational extreme in which the long-wavelength peak of the emission feature has disappeared entirely.

4. The ${ }^{12} \mathrm{CO}$ and ${ }^{13} \mathrm{CO} J=1-0$ line emissions are unresolved with our $\sim 8^{\prime \prime}$ beam. The integrated ${ }^{12} \mathrm{CO} J=1-0$ line consists of a strong central component, implying the presence of a molecular shell expanding at $V_{e, \mathrm{AGB}} \sim 15 \mathrm{~km} \mathrm{~s}^{-1}$, and a weak, broad component with FWZI of $\sim 63 \mathrm{~km} \mathrm{~s}^{-1}$. The spatial peaks of the red and blue emission components in the broad velocity component are separated by about $3.4^{\prime \prime}$ along the long axis of the nebula and represent emission from a fast bipolar outflow. An unresolved, $3.6 \pm 0.8 \mathrm{mJy}$ central continuum emission source is also detected.

5. Using a spherical shell model, we have fitted the core emission profile from our observations and from additional ones for the $J=2-1$ and 4-3 lines reported in the literature, using a least-squares minimization procedure. We find, with the inner and outer radii of the shell constrained to lie within their observationally inferred ranges, that all the data can be fitted with $T_{\text {rot }}=14.5-21 \mathrm{~K}$ and a shell mass $\gtrsim 0.4 M_{\odot}$, since all ${ }^{12} \mathrm{CO}$ lines are optically thick. The average AGB mass-loss rate, $\dot{M} \gtrsim$ $2 \times 10^{-4} M_{\odot} \mathrm{yr}^{-1}$, and the corresponding mass-ejection timescale is about $2000 \mathrm{yr}$. The ${ }^{13} \mathrm{C} /{ }^{12} \mathrm{C}$ ratio is less than or about 0.1 .

6. The observed $2.6 \mathrm{~mm}$ continuum as well as the published submillimeter $(850 \mu \mathrm{m})$ continuum fluxes are far in excess of those predicted by a recently published detailed dust-emission model of the full SED of I19475. In order to explain this longwavelength excess, we need to include emission from a substantial mass $\left(0.045 M_{\odot}\right)$ of very large $(\gtrsim 2.6 \mathrm{~mm})$, cool $(30 \mathrm{~K})$ grains, located at a radial distance of about $1.3 \times 10^{17} \mathrm{~cm}$.

7. Combining our estimates of the total mass of circumstellar material with the typical mass of a post-AGB star, we find that the mass of the main-sequence progenitor of I19475's central star was at least $2.5 M_{\odot}$.

This research has made use of the USNOFS Image and Catalogue Archive operated by the United States Naval Observatory, Flagstaff Station (http://www.nofs.navy.mil/data/fchpix). R. S. and M. M. thank NASA for partially funding this work by a NASA LTSA award (399-20-40-06); R. S. also received partial support for this work from NASA ADP award (399-20-00-08) and HST/GO awards (GO-09463.01-A and GO-09801.01-A) from the Space Telescope Science Institute, which is operated by the AURA, Inc., under NASA contract NAS5-26555). C. S. C. is partially funded for this work by National Science Foundation grant 9981546 to Owens Valley Radio Observatory, the Spanish MCyT under project AYA2003-2785, and the Astroscam project (Ref: S-0505 ESP-0237).
Balick, B., \& Frank, A. 2002, ARA\&A, 40, 439

Blackman, E. G., Frank, A., \& Welch, C. 2001, ApJ, 546, 288

Bowers, P. F., Johnston, K. J., \& Spencer, J. H. 1983, ApJ, 274, 733

Bujarrabal, V., Castro-Carrizo, A., Alcolea, J., \& Sánchez Contreras, C. 2001, A\&A, 377, 868
REFERENCES

Condon, J. J., Cotton, W. D., Greisen, E. W., Yin, Q. F., Perley, R. A., Taylor, G. B., \& Broderick, J. J. 1998, AJ, 115, 1693

Gledhill, T. M., Bains, I., \& Yates, J. A. 2002, MNRAS, 332, L55

Gledhill, T. M., Chrysostomou, A., Hough, J. H., \& Yates, J. A. 2001, MNRAS, 322, 321 
Goodrich, R. W. 1991, ApJ, 376, 654

Hakkila, J., Myers, J. M., Stidham, B. J., \& Hartmann, D. H. 1997, AJ, 114, 2043

Hrivnak, B. J., \& Bieging, J. H. 2005, ApJ, 624, 331

Hrivnak, B. J., Langill, P. P., Su, K. Y. L., \& Kwok, S. 1999, ApJ, 513, 421

Jura, M., Webb, R. A., \& Kahane, C. 2001, ApJ, 550, L71

Klochkova, V. G., Panchuk, V. E., \& Tavolzhanskaya, N. S. 2002, Astron. Lett., 28,49

Likkel, L., Forveille, T., Omont, A., \& Morris, M. 1991, A\&A, 246, 153

Manchado, A., Stanghellini, L., \& Guerrero, M. A. 1996, ApJ, 466, L95

Mastrodemos, N., \& Morris, M. 1998, ApJ, 497, 303 1999, ApJ, 523, 357

Matt, S., Frank, A., \& Blackman, E. 2004, in ASP Conf. Ser. 313, Asymmetrical Planetary Nebulae III, ed. M. Meixner, J. H. Kastner, B. Balick, \& N. Soker (San Francisco: ASP), 34

Olofsson, H., Carlstrom, U., Eriksson, K., Gustafsson, B., \& Willson, L. A. 1990, A\&A, 230, L13

Ossenkopf, V. 1991, A\&A, 251, 210

Pollack, J. B., Hollenbach, D., Beckwith, S., Simonelli, D. P., Roush, T., \& Fong, W. 1994, ApJ, 421, 615
Sahai, R. 1990, ApJ, 362, 652

2002, Astrophys. Space Sci. Libr. 265, 53

2004, in ASP Conf. Ser. 313, Asymmetrical Planetary Nebulae III, ed. M. Meixner, J. H. Kastner, B. Balick, \& N. Soker (San Francisco: ASP), 141 Sahai, R., \& Sánchez Contreras, C. 2004, in ASP Conf. Ser. 313, Asymmetrical Planetary Nebulae III, ed. M. Meixner, J. H. Kastner, B. Balick, \& N. Soker (San Francisco: ASP), 34

Sahai, R., Sánchez Contreras, C., \& Morris, M. 2005, ApJ, 620, 948

Sahai, R., \& Trauger, J. T. 1998, AJ, 116, 1357

Sahai, R., Young, K., Patel, N., Sánchez Contreras, C., \& Morris, M. 2006, ApJ, 653, 1241

Sahai, R., Zijlstra, A., Bujarrabal, V., \& Te Lintel Hekkert, P. 1999, AJ, 117, 1408 Sahai, R., Zijlstra, A., Sánchez Contreras, C., \& Morris, M. 2003, ApJ, 586, L81 Sarkar, G., \& Sahai, R. 2006, ApJ, 644, 1171 (SS06)

Sánchez Contreras, C., Bujarrabal, V., Castro-Carrizo, A., Alcolea, J., \& Sargent, A. 2006, ApJ, 643, 945 (SCetal06)

Sánchez Contreras, C., Le Mignant, D., Sahai, R., Gil de Paz, A., \& Morris, M. 2007, ApJ, 656, 1150

Sopka, R. J., Hildebrand, R., Jaffe, D. T., Gatley, I., Roellig, T., Werner, M., Jura, M., \& Zuckerman, B. 1985, ApJ, 294, 242 